\title{
Interfacial Instability in Electrified Plane Couette Flow
} STEFAN M ÄHLMAN N AND DEMETRIOS T. PAPAGEORGIOU ${ }^{1,2}$

\footnotetext{
${ }^{1}$ Department of Mathematical Sciences and Center for Applied Mathematics and Statistics, New Jersey Institute of Technology, Newark, New Jersey 07102. USA

${ }^{2}$ Department of Mathematics and Department of Chemical Engineering, Imperial College London, London SW7
} $2 \mathrm{AZ}, \mathrm{UK}$.

(Received 2nd August 2010)

The dynamics of a plane interface separating two sheared, density and viscosity matched fluids in the vertical gap between parallel plate electrodes are studied computationally. A Couette profile is imposed onto the fluids by moving the rigid plates at equal speeds in opposite directions. In addition, a vertical electric field is applied to the shear flow by impressing a constant voltage difference on the electrodes. The stability of the initially flat interface is a very subtle balance between surface tension, inertia, viscosity, and electric field effects. Under unstable conditions, the potential difference in the fluid results in an electrostatic pressure that amplifies disturbance waves on the two-fluid interface at characteristic wave lengths. Various mechanisms determining the growth rate of the most unstable mode are addressed in a systematic parameter study. The applied methodology involves a combination of numerical simulation and analytical work. Linear stability theory is employed to identify unstable parametric conditions of the perturbed Couette flow. Particular attention is given to the effect of the applied electric field on the instability of the perturbed two-fluid interface. The normal mode analyses are followed up by numerical simulations. The applied method relies on solving the governing equations for the fluid mechanics and the electrostatics in a one-fluid approximation by using a finite-volume technique combined with explicit tracking of the evolving interface. The numerical results confirm those of linear theory and, furthermore, reveal a rich array of dynamical behavior. The elementary fluid instabilities are finger-like structures of interpenetrating fluids. For weakly unstable situations a single fingering instability emerges on the interface. Increasing the growth rates causes the finger to form a drop-like tip region connected by a long thinning fluids neck. Even more striking fluid motion occurs at higher values of the electric field parameter for which multiple fluid branches develop on the interface. For a pair of perfect 
dielectrics the vertical electric field was found to enhance interfacial motion irrespective of the permittivity ratio, while in leaky dielectrics the electric field can either stabilize or destabilize the interface, depending on the conductivity and permittivity ratio between the fluids.

\section{Introduction}

Recent progress in the design and utilization of micro-fluidic devices for fluid transport has found many applications, ranging from the life-sciences industry for pharmaceuticals and biological medicine (drug design, delivery and detection, and diagnostic devices) to industrial applications of combinatorial synthesis and polymer extrusion. Other areas of applications include aerospace and automotive industries, and microreaction engineering. The most important new features introduced by the small length scales of micro-fluidic devices are the significant role of interfacial forces (adhesive forces, electric fields, van der Waals interactions), surface roughness, energy dissipation, and fluidic resistance.

Micro-fluidic flows with Reynolds numbers of order unity or smaller are often stable and governed by viscous dissipation. As the Reynolds number increases the presence of inertia introduces a non-linearity to the problem which is a source of flow instabilities. For example, devices for mixing and dispensing benefit from flow instabilities, particularly due to the absence of turbulence at low Reynolds numbers, and it has been suggested (El Moctar, Aubry \& Batton 2003, Uguz, Ozen \& Aubry 2008, Uguz \& Aubry 2008 - see also the experiments of Ozen et al. 2006a) that electric fields may be useful in enhancing instabilities in such flows even at small Reynolds numbers.

The presence of multiple phases poses major challenges in both proper design and reliable operation of flow devices. Typically, multi-fluidic systems are susceptible to hydrodynamic instabilities, blockages, and pressure and temperature fluctuations - see for example Charles, Govier \& Hodgson (1961), Hagerdon, Martyn \& Douglas (2004). Hence, the establishment of waves on an originally flat interface separating different fluid phases has a strong impact on the overall performance of the assembly. For this reason, the control and use of such fluid devices requires a clear insight into the conditions at which waves form as well as their ultimate non-linear evolution. 
The stability of two superposed viscous fluids in plane channel flow has attracted considerable theoretical and practical interest over many years. In a pioneering study Yih (1967) examined the linear stability of superposed Couette and plane Poiseuille flows using a long-wave approach. He showed that viscosity stratification can generate an interfacial instability (the interfacial mode) at any Reynolds number. In addition to instability caused by viscosity stratification, hydrodynamic instability can also arise from density stratification, curvature of the velocity profile or from shear effects in one of the phases. In addition to the interfacial mode, Hooper \& Boyd (1983) found a viscous-inertial dominated instability mechanism in two-layer Couette flow, primarily at short wave lengths. Two-layer Poiseuille flow also supports a shear-type instability, closely resembling the modes of the single-phase Poiseuille flow. Renardy (1985) carried out a full linear stability analysis and revealed the existence of unstable parameter regions in channel flow of two stratified viscous fluids which had been missed out by previous long or short wave asymptotic analyses. There exists a large literature on the linear stability of two-layer flow in the absence of electric fields; the reader is referred to Renardy (1987), Yiantsios et al. (1988), Hooper (1989), and the references therein.

The electrohydrodynamic (abbreviated EHD hereafter) instability of superposed, immiscible, viscous fluids has several applications particularly in ink-jet printing, lithography, and biological colloidal physics. The electrohydrodynamic activity of the fluid system considered in this article rests on the presence of mobile charges that move locally under the influence of an external electric field and migrate onto the interface inducing polarization forces. The strength of the electric fields in the dielectrics depends on the polarizability of the fluids, expressed in the dielectric constants $\epsilon$. With a mismatch of the polarizability between the two fluids, the electric field exerts a force on the charges at the fluid interface. This electrostatic force can either be normal or tangential to the interface depending on the conductivity and dielectric properties of the fluids. For perfect dielectrics and conductors, the resulting force acts perpendicular to the interface, thus pushing the interface in the direction with the lower dielectric constant. The idealization of non-conducting perfect dielectric fluids provides a useful leading order approximation. However, most real non-conducting fluids do not behave as perfect insulators, irrespective of how small their conductivity is. Even a small conductivity allows electric charges to reach the interface and form a diffuse charge layer there. Given the apparent importance of finite conductivities in technological applications we adopt the leaky dielectric model 
developed by Taylor \& Melcher (1969). In the Taylor-Melcher model dielectric fluids have small but finite conductivities and the change of the charge density by fluid motion is assumed to be small and thus negligible in the conservation of charge equation. For this reason, the electric field can be computed from the charge conservation equation governing the electric potential. The Taylor-Melcher model allows for redistribution and accumulation of free charges at interfaces and thus predicts tangential stresses due to charge buildup there (see Saville 1997). It has been established that tangential electric stresses can significantly affect the stability of multi-fluidic systems as they induce convective flows and fluid deformations that cannot be generated by distributed body forces.

It is well known that surface polarization forces can lead to the instability of plane interfaces between two viscous fluids in a vertical uniform electrostatic field (Melcher \& Smith 1969). The case of two superposed conducting or non-conducting fluids was examined by Melcher \& Schwarz (1968). They considered a constant electric field applied in the plane of the undisturbed interface and analyzed the linear stability of the twophase systems showing that the influence of the electric field is a dispersive regularization of short waves. Melcher \& Schwarz (1968) also pointed out that the electric field produces net surface forces due to the polarization that tend to disrupt the interface and they studied the effect of an electric field on the linear stability separating two non-conducting dielectric fluids of infinite extent. They established that the electric field stabilizes and increases the propagation speed of surface waves. This behavior continues into the fully non-linear regime as discovered in the computations of Papageorgiou \& Vanden-Broeck (2004). The stability of Couette-Poiseuille flow in the presence of a vertical electric field has been studied by Ozen et al. (2006b), Li et al. (2007), Uguz et al. (2008) and Uguz \& Aubry (2008) when the field is either vertical or horizontal to the undisturbed interface. Both perfect and leaky dielectric models are considered and it is shown that vertical fields destabilize perfect dielectric fluid pairs while for leaky dielectrics the situation is more complicated in that there exist parameter ranges that depend on the permittivity and conductivity ratios for which the flow can be stabilized. One of the main objectives of the present study is to extend such linear theories into the non-linear regime at arbitrary Reynolds numbers.

Of interest to the present work are electrokinetic flows in microchannels in the presence of conductivity gradients. Lin et al. (2004) consider instabilities (theoretically and experimentally) in a rectangular channel 
driven by an electric field which is perpendicular to a conductivity stratification gradient, building on earlier work by Baygents \& Baldessari (1998). Additional studies including the transition from convective to absolute instability as well as work on colloidal suspensions can be found in Chen et al. (2005), Lin (2009), Posner \& Santiago (2006), and Navaneetham \& Posner (2009). The physical problem in the cited studies is typically a single-phase flow driven by spatio-temporal gradients in electrical conductivity in the absence of sharp interfaces.

The present study is partly motivated by the micro-fluidic experiments of Ozen et al. (2006a) at fairly small shear rates characterized by Reynolds numbers of order $\mathcal{O}\left(10^{-2}\right)$. They produced highly stable two-fluid Poiseuille flows in microchannels and studied their stability under electric fields perpendicular to the meanflow direction. Ozen et al. showed experimentally that electrohydrodynamically induced instabilities can lead to a separation of the initially stable liquid layers into droplets of one phase encapsulated in the second immiscible phase. This is achieved by first producing an alternating series of liquid plugs of one fluid followed by the second one, which then relax to Bretherton-like droplets when they are swept by the flow outside the region where the field is acting. What is most striking, perhaps, is that this non-linear phenomenon is periodic and extremely robust thus producing a monodisperse distribution of droplets. In a related theoretical work, Mählmann \& Papageorgiou (2009), consider the electrification of such an array of droplets of one fluid in another under shear. Direct numerical simulations are carried out (using level-set methods) that establish the role of the electric field to enhance stretching of the drops and indeed destabilize steady deformed droplet shapes into ones that are stretched indefinitely. Thaokar \& Kumaran (2005) studied theoretically the effect of vertical electric fields on the stability of the interface between two perfect and leaky dielectric fluids in the zero Reynolds number limit. Using weakly non-linear and boundary integral analysis they focused on the nature of the initial bifurcation, which cannot be addressed by the linear stability theory. Tomar et al. (2007) addressed this problem at arbitrary Reynolds number using a coupled level set and volume-of-fluid method. Moreover, Craster \& Matar (2005) derived a coupled system of evolution equations to investigate the stability of the interface between two thin leaky dielectric fluid layers. By performing linear and nonlinear stability analyses they found that decreasing the thickness ratio destabilizes the interface, giving rise to periodic structures of decreasing wavelength. 
There exists a large variety of computational models for moving interfaces, grossly categorized into interface capturing and interface tracking methods. In interface capturing methods such as level-set (Osher \& Sethian 1988) and phase-field methods (Anderson et al. 1998), the interface is implicitly represented by a contour of a scalar function, whereas interface tracking methods (examples include boundary integral, Pozrikidis 2001, volume-of-fluid, Hirt \& Nichols 1981, front-tracking, Glimm et al. 1998, Unverdi \& Tryggvason 1992, immersed boundary, Peskin 2002, and immersed interface methods, LeVeque \& Li 1994, LeVeque \& Lee 2003) use a Lagrangian approach to track the interface. The basic idea of the front-tracking approach is the use of two different grids. The fluid equations are solved on a fixed Eulerian grid, whereas Lagrangian coordinates are employed to explicitly track the evolving interface and to accurately compute surface forces. Front-tracking methods have shown several advantages in the computation of physical problems such as the study of fluid interface instabilities (see Du et al. 2005). For this reason, the front-tracking technique will be applied to the physical problem considered in this study.

The main goal of the present work is to elucidate the physical mechanisms that affect the interfacial instability in electrified two-layer Couette flow at arbitrary Reynolds numbers and to follow the dynamics into the non-linear regime in order to allow interfacial touchdown. Our methodology involves a combination of numerical simulation and analytical work. Linear stability theory is employed to identify unstable parametric conditions of the perturbed two-layer Couette flow, and is followed up by numerical simulations of the transient response of the system to small amplitude disturbances. The computational study addresses the combined effects of electric fields, surface tension, and inertia on the interfacial dynamics and covers pairs of perfect and leaky dielectric fluids. To identify the linear regime we compare growth rates of the evolving instability computed from the numerical data with theoretical predictions based on the linear stability theory. Such disturbances are then followed into the fully non-linear regime computationally up to times when (if) the interface touches one of the channel walls.

The remainder of this article unfolds as follows: In Section 2 we outline the physical problem and formulate the governing equations for the fluid mechanics and the electrostatics. The solution of the coupled system of equations yields transient simulations for the motion of the perturbed interface in perfect or leaky dielectric fluids. Furthermore, we summarize the linear stability equations and describe the applied technique to solve 


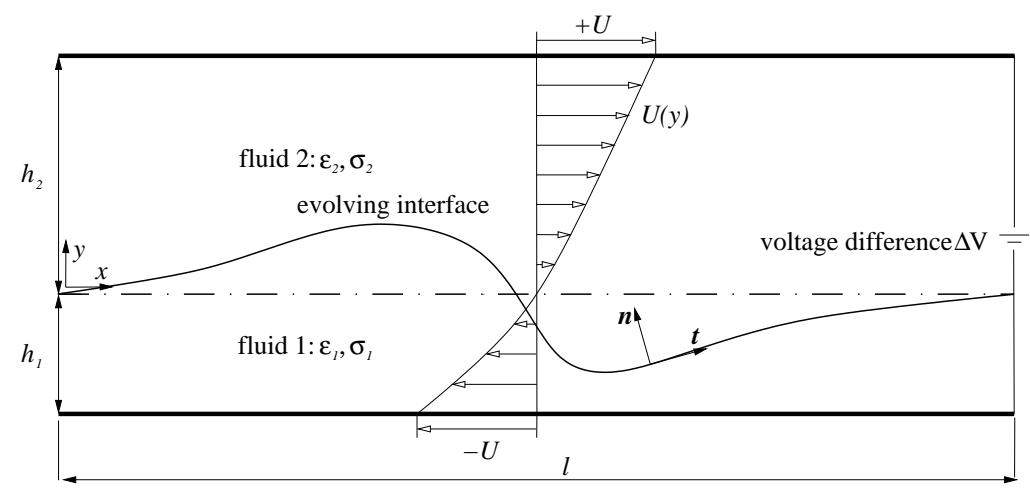

FIGURE 1. Schematic representation of a evolving interface in plane two-layer Couette flow under the influence of a vertical electric field.

the generalized eigenvalue problem. Section 3 presents results of the numerical simulations and comparisons with the linear stability analysis for a wide number of process parameters. In Section 4 we summarize our conclusions.

\section{Problem Formulation}

\subsection{Governing Equations and Method of Solution}

In this section we will first present the fundamental equations governing the dynamics of a sheared two-fluid interface including electric field effects. A schematic representation of the problem of interest is depicted in Figure 1. We consider a system of two superposed incompressible, density- and viscosity-matched fluids between two rigid horizontal plates. The fluids are immiscible and separated by a deformable sharp interface. The lower/upper fluids are referred to as fluids 1 and 2, respectively; fluid $j(j=1,2)$ has undisturbed thickness $h_{j}$, dielectric permittivity $\epsilon_{j}$, and electric conductivity $\sigma_{j}$. A Couette profile is generated by moving the parallel plates confining the fluids at equal speeds $U$ in opposite directions. In addition, a uniform electric field is imposed on the system by applying a constant voltage potential difference $\Delta V=V_{0}$ in the vertical gap between the plates. Gravitational effects are neglected in this study, which is a useful assumption in the regime of micro-fluidic flow scales. The no-slip/no-penetration condition is imposed at the rigid plates. The infinite horizontal extent of the flow is modeled by a periodic representation, where the length of the computational domain $l$ represents one wavelength of the imposed disturbance. The mathematical description of the time-dependent interfacial dynamics requires simultaneous solution of the fluid mechanics 
equations and the equations of electrostatics. Introducing dimensionless variables that are constructed using the material properties of the lower fluid as reference values, the velocity scale $U$ and the time scale $h_{1} / U$, we find that the problem is characterized by the Reynolds number $R e$, the surface tension parameter $T$, and the electric field parameter $E_{w}$ defined as follows

$$
R e=\frac{\rho_{1} U h_{1}}{\mu_{1}}, \quad T=\frac{\gamma}{\rho_{1} U^{2} h_{1}}, \quad E_{w}=\frac{\epsilon_{1} \epsilon_{0} V_{0}^{2}}{\rho_{1} U^{2} h_{1}^{2}}
$$

where $U$ is the speed of the plates of the Couette device, $\rho$ is the density, $\mu$ is the dynamic viscosity, $\gamma$ is the coefficient of surface tension (assumed constant), and $\epsilon_{0}$ is the permittivity of free space $\left(\epsilon_{0}=8.85 \times 10^{-12}\right.$ $\mathrm{C} / \mathrm{Vm})$. We continue by formulating the two sets of governing equations pertaining to the dynamics of the two-layer Couette flow with the fluid motion of each phase described by a single set of conservation laws. Furthermore, localized forces that arise due to the singular nature of the two-fluid interface are treated by adding appropriate source terms to the momentum equations. It follows that the dimensionless governing equations for a quasi one-fluid flow with spatially varying material properties read

$$
\begin{aligned}
\boldsymbol{\nabla} \cdot \boldsymbol{u} & =0 \\
\frac{\partial \rho \boldsymbol{u}}{\partial t}+\nabla \cdot \rho \boldsymbol{u} \boldsymbol{u} & =-\nabla p+R e^{-1} \nabla \cdot\left(2 \mu \boldsymbol{D}_{u}\right)+\boldsymbol{F}_{s} .
\end{aligned}
$$

Here, $\boldsymbol{u}$ is the velocity vector, $p$ is the pressure and $\boldsymbol{D}_{u}=\frac{1}{2}\left(\boldsymbol{\nabla} \boldsymbol{u}+\boldsymbol{\nabla} \boldsymbol{u}^{T}\right)$ is the deformation tensor. $\boldsymbol{F}_{s}$ represents the singular interface force, which is composed of two parts in our study, i) the surface tension force $\boldsymbol{F}_{s}^{\gamma}$, and ii) the electrostatic force density $\boldsymbol{F}_{s}^{e}$. Here, surface tension is represented by a distribution of singularities along the interface

$$
\boldsymbol{F}_{s}^{\gamma}=T \int_{\partial \Omega} \kappa \boldsymbol{n} \delta\left(\boldsymbol{x}-\boldsymbol{x}_{f}\right) d s,
$$

where $\kappa$ is twice the mean curvature of the interface, $\delta$ is a three-dimensional delta-function constructed as a tensor product of one-dimensional Dirac delta functions, $\boldsymbol{n}$ is a unit vector normal to the interface, $\boldsymbol{x}$ is the point at which the equations are evaluated and $\boldsymbol{x}_{f}$ is the position of the front. Following Shin \& Juric (2002), the discrete numerical implementation of the surface integral (2.4) onto the fixed Eulerian grid is approximated in the form of a sum over all interface elements

$$
\sum_{k} \boldsymbol{f}_{k} \delta\left(\boldsymbol{x}-\boldsymbol{x}_{k}\right) \Delta s_{k}
$$


where $\Delta s_{k}$ denotes the average of the straight line distances from point $\boldsymbol{x}_{f, k}$ to the two neighboring points $\boldsymbol{x}_{f, k+1}$ and $\boldsymbol{x}_{f, k-1}$, and $\boldsymbol{f}_{k}$ is the surface tension force given by the Serret-Frenet formula. To transfer the front singularities to the fixed grid the sharp delta functions are approximated by smooth functions with compact support on the grid. More precisely, we used the interpolation function proposed by Peskin (1977) to interpolate the velocities and to distribute the surface forces onto the grid. The standard diffuseinterface approximation is employed, where the discontinuous material fields $\boldsymbol{q}(\boldsymbol{x})$ are replaced by smoothed distributions defined in terms of a smooth scalar function $\varphi(\boldsymbol{x})$. This function takes the value of zero in fluid region 1, the value of unity in region 2, and undergoes a gradual transition across the interface. The permittivity and conductivity field $\boldsymbol{q}^{s}=(\epsilon, \sigma)^{s}$ are approximated using the linear interpolation

$$
\boldsymbol{q}^{s}(\boldsymbol{x})=\boldsymbol{q}_{1}+\left(\boldsymbol{q}_{2}-\boldsymbol{q}_{1}\right) \varphi(\boldsymbol{x}) .
$$

By smoothing the delta function and taking the divergence of the resulting equation, we derive a Poisson equation for the smooth function $\varphi(\boldsymbol{x})$. The forcing term on the right-hand side represents the divergence of a nearly singular vector field distributed along the interface.

Next we consider the electric field problem and provide a formulation that is later utilized in a diffuse interface numerical method. The starting point is the system of Maxwell's equations in regions 1 and 2 (e.g. Jackson 1962). In the problems of interest here (either DC fields or AC fields at frequencies in the KHz range and system lengths of the order of a meter or smaller), induced magnetic fields are small and can be ignored. The equations in each region become (subscripts are suppressed for clarity)

$$
\begin{gathered}
\boldsymbol{\nabla} \cdot(\epsilon \boldsymbol{E})=q, \\
\boldsymbol{\nabla} \times \boldsymbol{E}=0, \\
\frac{\partial q}{\partial t}+\boldsymbol{\nabla} \cdot \boldsymbol{J}=0,
\end{gathered}
$$

where $\boldsymbol{E}$ is the electric field, $q(\boldsymbol{x}, t)$ is the local charge density, $\boldsymbol{J}=\sigma \boldsymbol{E}+q \boldsymbol{u}$ is the current density, and $\epsilon$ and $\sigma$ denote the permittivity and conductivity constants in the specified region. Equation (2.7) is Gauss's law, equation (2.8) states that the electric field is irrotational since magnetic induction is negligible, and equation (2.9) is a volume charge density conservation equation. Eliminating $\boldsymbol{J}$ in favor of $q$ ((2.7) is used also), casts 
(2.9) into

$$
\frac{\partial q}{\partial t}+\boldsymbol{u} \cdot \nabla q+\frac{\sigma}{\epsilon} q=0
$$

which simplifies considerably in a Lagrangian frame. Typical values (e.g. de-ionized water) provide $\frac{\sigma}{\epsilon} \sim$ $10^{5} \mathrm{~s}^{-1}$ and hence a fast relaxation time-scale compared to the hydrodynamic one, which removes charges from the bulk since $q \rightarrow 0$ exponentially fast. To a good approximation, therefore, all charges will reside on the interface and in regions 1 and 2 we can take $q \equiv 0$. It remains to find the electrically induced force in the Navier-Stokes equations (2.3). For both perfect and leaky dielectric fluids the singular force density $\boldsymbol{F}^{s}$ is computed from the divergence form of the Maxwell stress tensor $\boldsymbol{T}^{M}$

$$
\boldsymbol{F}^{e}=E_{w} \nabla \cdot \boldsymbol{T}^{M}
$$

Following Landau \& Lifshitz (1960) and assuming linear isotropic media, the components of the Maxwell stress tensor $T_{i j}^{M}$ read

$$
T_{i j}^{M}=\epsilon \epsilon_{0} E_{i} E_{j}-\frac{1}{2} \epsilon \epsilon_{0}\left[1-\frac{\rho}{\epsilon} \frac{\partial \epsilon}{\partial \rho}\right] \boldsymbol{E} \cdot \boldsymbol{E} \delta_{i j} .
$$

The first term on the right of equation (2.12) represents the electrophoretic component that originates from Coulomb stresses upon free charges and the second term corresponds to dielectrophoric stresses (electrostrictive stresses are ignored since we assume that $\frac{\partial \epsilon}{\partial \rho}=0$ ). We also note the following equivalent form for the electrohydrodynamic force density $\boldsymbol{F}^{e}$ (this is easily verified by starting from (2.11) and using Gauss's law (2.7)

$$
\boldsymbol{F}_{s}^{e}=q \boldsymbol{E}-\frac{1}{2}(\boldsymbol{E} \cdot \boldsymbol{E}) \boldsymbol{\nabla} \epsilon
$$

This form of force density in single-phase flows with variable conductivities but constant permittivities, so that only the first term on the right hand side of (2.13) enters, has been used in a series of studies on electrokinetic instabilities by Chen et al. (2005), Lin et al. (2004), Lin (2009), Posner \& Santiago (2006) and Navaneetham \& Posner (2009). In what follows we discuss the individual cases of perfect and leaky dielectric fluids. We note that in all cases equation (2.8) is satisfied by introducing a voltage potential $V$ so that $\boldsymbol{E}=-\nabla V$.

When the fluids are perfect dielectrics (insulators), the system (2.7)-(2.9) reduces to the single quasi 
one-fluid equation

$$
\nabla \cdot(\epsilon \nabla V)=0,
$$

where the permittivity $\epsilon$ is variable across the interface according to (2.6) and its mollified version. Once the electric field is determined throughout the domain, the electric force (2.11) (or equivalently (2.13)) in the Navier-Stokes equations drives the electrohydrodynamic instabilities.

When the conductivities are finite we model the problem using the leaky dielectric model. Noting the additional fast relaxation time limit mentioned earlier we observe that the charge density $q$ is non-zero on the interface alone. The quasi one-region equation that emerges is

$$
\nabla \cdot(\sigma \nabla V)=0,
$$

where again $\sigma$ varies across the interface - see (2.6). Solution of (2.15) for $V$ completely determines the charge density in the vicinity of the interface and the resulting electric force (2.11) that enters into the Navier-Stokes equations. Different but completely equivalent versions of this force appear in the literature; for example in the single-phase electrokinetic studies with variable conductivity of Lin (2009) and references therein, the force $\boldsymbol{F}_{s}^{e}$ appearing in (2.3) is seen to be $\nabla^{2} V \nabla V$. This arises from the term $q \boldsymbol{E}$ in (2.13) when $q$ is eliminated in favor of $V$ using the Gauss law (2.7), assuming constant permittivity and scaling velocities on their electro-viscous magnitude. In addition, it is easily seen from Gauss's law coupled with (2.15) that when the permittivity is constant as assumed in the single-phase electrokinetic studies, the identity $q=(\epsilon / \sigma) \nabla \sigma \cdot \nabla V$ emerges. In our case $\epsilon$ varies also in the vicinity of the interface and the appropriate value is $q=(\epsilon / \sigma) \nabla \sigma \cdot \nabla V-\nabla \epsilon \cdot \nabla V$ which can be used in (2.13) to give an alternative derivation of the electric density force used in our computations.

The EHD equations are discretized on a fixed, staggered Cartesian grid. The moving interface is represented by an ordered list of implicitly connected particles. In order to keep the interface sharp the marker points are advected in a Lagrangian manner. Since the interface propagates with the fluid flow, the velocity of the marker points has to be interpolated from the fixed grid. Once the velocity of each marker point on the interface has been found, its new position is determined by integration. As the interface deforms and stretches during the simulation, the resolution of some parts of the interface can become inadequate, while 
elements are accumulated in other parts. To ensure adequate spatial resolution and to maintain regularity and stability of the interface representation at all times, marker points are dynamically added and deleted during the computation. When new marker points are introduced, their position is computed by fourth-order interpolation with respect to an approximate arc length. At each time step, after the interface position has been updated, the discontinuous material fields are reconstructed by integration of the smooth grid-delta function. The momentum equations are discretized using a conservative, second-order centered difference scheme for the spatial variables and a second-order Adams-Bashforth time integration scheme. Then, singular forces are added to the nodal values of the momentum equations. Finally, an intermediate velocity field is calculated from the momentum equations which is then projected onto the space of divergence-free vector fields. The solution of the discretized Poisson equations required at each time step is carried out using a multi-grid method (see Mählmann \& Papageorgiou 2009 also). The formulation used is analogous to that of Unverdi \& Tryggvason (1992), and notable differences include the multi-grid solvers utilized for the solution of the Poisson problems for the pressure and electric potential, along with the necessary modifications needed to incorporate the electric stress in the momentum equations, as described above.

\subsection{Linear Stability Theory}

The electrohydrodynamic stability of the two-layer Couette flow is analyzed by solving an eigenvalue problem resembling the Orr-Sommerfeld one. Results of linear studies have been reported elsewhere (Ozen et al. 2006b, Li et al. 2007, Uguz et al. 2008, Uguz \& Aubry 2008) and for completeness, in what follows we give a brief description of our numerical work since the results are used to test the validity of linear theory. Introducing a perturbation streamfunction $\psi^{(j)}(x, y, t)$ we write $\left.\left(u^{(j)}, v^{(j)}\right)=\left(U_{j}(y), 0\right)+\eta\left(\tilde{u}^{(j)}, \tilde{v}^{(j)}\right)\right)$ where $|\eta| \ll 1$ is a linearization parameter and $\left(\tilde{u}^{(j)}, \tilde{v}^{(j)}\right)=\left(\partial \psi^{(j)} / \partial y,-\partial \psi^{(j)} / \partial x\right)$, the streamfunction $\psi$ is expressed in terms of normal modes

$$
\psi(x, y, t)=\hat{\phi}(y) e^{i k(x-c t)}+\text { c.c. }
$$

where c.c. denotes complex conjugate, $\hat{\phi}(y)$ is the complex amplitude, $k$ is the dimensionless real wave number, and $c=c_{r}+i c_{i}$ is the complex wave speed. The real part $c_{r}$ is the phase velocity of the wave, while $k c_{i}$ is its growth rate (the flow is unstable if $c_{i}>0$, stable if $c_{i}<0$, and neutrally stable when $c_{i}=0$ ). 
Linearizing the Navier-Stokes equations, eliminating the pressure, and using the definition (2.16), yields the Orr-Sommerfeld system

$$
\begin{gathered}
\left(\frac{d^{2}}{d y^{2}}-k^{2}\right)^{2} \phi^{(1)}=i k R e_{1}\left\{\left(U_{1}-c\right)\left(\frac{d^{2}}{d y^{2}}-k^{2}\right)-\frac{d^{2} U_{1}}{d y^{2}}\right\} \phi^{(1)}, \quad-h_{1}<y<0, \\
\left(\frac{d^{2}}{d y^{2}}-k^{2}\right)^{2} \phi^{(2)}=\left(i k R e_{1} \rho / m\right)\left\{\left(U_{2}-c\right)\left(\frac{d^{2}}{d y^{2}}-k^{2}\right)-\frac{d^{2} U_{2}}{d y^{2}}\right\} \phi^{(2)}, \quad 0<y<h_{2} .
\end{gathered}
$$

For generality we have implemented the linear stability work for arbitrary density and viscosity ratios and these appear in (2.18) above as the new parameters $\rho=\rho_{2} / \rho_{1}$ and $m=\mu_{2} / \mu_{1}$.

The boundary conditions expressing no-slip and no-penetration at the walls read

$$
\phi^{(1)}=\frac{d \phi^{(1)}}{d y}=0 \text { at } y=-h_{1} \quad \text { and } \quad \phi^{(2)}=\frac{d \phi^{(2)}}{d y}=0 \text { at } y=h_{2} \text {. }
$$

In addition to equations (2.17), (2.18), four conditions are necessary to satisfy the continuity of the velocity and stress components, both in the tangential and normal direction. These read, at $y=0$,

$$
\begin{array}{r}
\phi^{(1)}=\phi^{(2)}, \\
\frac{d \phi^{(1)}}{d y}+\frac{d U_{1}}{d y} \phi^{(1)} / c=\frac{d \phi^{(2)}}{d y}+\frac{d U_{2}}{d y} \phi^{(2)} / c+i k R e E_{w} K^{T} \hat{s}, \\
\left(\frac{d^{2}}{d y^{2}}+k^{2}\right) \phi^{(1)}+\frac{d^{2} U_{1}}{d y^{2}} \phi^{(1)} / c=\mu\left[\left(\frac{d^{2}}{d y^{2}}+k^{2}\right) \phi^{(2)}+\frac{d^{2} U_{2}}{d y^{2}} \phi^{(2)} / c\right], \\
\left(\frac{d^{2}}{d y^{2}}-3 k^{2}\right) \frac{d \phi^{(1)}}{d y}-i k \operatorname{Re}\left(c \frac{d \phi^{(1)}}{d y}+\frac{d U_{1}}{d y}\right)= \\
\left(\frac{d^{2}}{d y^{2}}-3 k^{2}\right) \frac{d \phi^{(2)}}{d y}-i \rho k \operatorname{Re}\left(c \frac{d \phi^{(2)}}{d y}+\frac{d U_{2}}{d y}\right)+i k \operatorname{Re}\left(\rho K^{F}-\left(k^{2} T \phi^{(2)} / c+E_{w} K^{N}\right) \hat{s}\right) .
\end{array}
$$

The amplitude of the interfacial perturbation $\hat{s}$ in (2.21)-2.23) is given by $\hat{s}=\phi^{(1)}(0) /(c-U(0))$, and we define $K^{F}, K^{N}, K^{T}$ to represent the effects of density stratification, and tangential and normal electric stresses in the conditions above,

$$
\begin{array}{r}
K^{F}=(c-U(0))\left(\frac{1}{\rho} \frac{d \phi^{(1)}}{d y}-\frac{d \phi^{(2)}}{d y}\right)-\phi(0)\left(\frac{1}{\rho} \frac{d U_{2}}{d y}-\frac{d U_{1}}{d y}\right) \\
K^{N}=\frac{1}{(n+\sigma)^{2}}\left[\left(\sigma^{2}-\epsilon\right)-(\sigma-1) \frac{\epsilon \tanh k n+\sigma^{2} \tanh k}{\tanh k n+\tanh \sigma k}\right] \\
K^{T}=\frac{(\sigma-1)\left(\epsilon-\sigma^{2}\right)}{(n+\sigma)^{2}} \frac{k}{\tanh k n+\tanh k}
\end{array}
$$

where $n=h_{1} / h_{1}$ denotes the ratio of the initial thicknesses of the fluids layers. A derivation of these conditions in the absence of the electric field can be found in Yih (1967), and we refer to Li et al. (2007) for details on the formulation of the electric stress conditions. We also note that the formulation above 
describes the general case of linear hydrodynamic stability of two superimposed fluids of different viscosities and densities in the absence of gravitational effects. Equations (2.17) and (2.18) together with the boundary and interface conditions (2.19) and (2.20)-(2.23) constitute an eigenvalue problem which is not self-adjoint and nontrivial solutions exist if and only if $c$ and $k$ satisfy a dispersion relation of the form

$$
\mathcal{D}\left(c, k, \rho, n, m, \mu, \epsilon, \sigma, R e, T, E_{w}\right)=0 .
$$

In order to determine the complex eigenvalue $c$, the eigenfunctions $\phi^{(j)}(y)$ are approximated by truncated Chebyshev expansions of the first kind

$$
\phi^{(j)}(y)=\sum_{n=0}^{N_{j}} a_{n}^{(j)} T_{n}^{i}(y)
$$

where $T_{n}^{i}(y)$ is the $n$th Chebyshev polynomial, $i$ denotes the $i$ th derivative with respect to $y, a_{n}^{j}$ are constants, and $N_{j}$ denote the number of collocation points in fluid layers 1 and 2, respectively. The derivatives of the eigenfunctions can be found by differentiating the Chebyshev polynomials in (2.28). Substitution of the Chebychev expansions into the eigenvalue problem (2.17)-(2.23) yields the generalized matrix-eigenvalue formulation

$$
\mathcal{A} \phi=c \mathcal{B} \phi
$$

which is linear and homogeneous in $a_{n}^{(j)}$. In this work, the matrices $\mathcal{A}, \mathcal{B}$ have been formulated using the D-2 scheme of Dongarra, Straughan \& Walker (1996). In addition, modified Chebychev differentiation matrices have been employed to ensure that the solution satisfies clamped boundary conditions at the walls (see Canuto et al. (1988) and Trefethen (2000) for details). The matrices $\mathcal{A}, \mathcal{B}$ are singular because some of the boundary and interface conditions do not contain the eigenvalue $c$. This singularity is handled by deflating the infinite eigenvalues using the standard QZ-algorithm to solve (2.29). Grid convergence has been checked by varying the number of collocation points $N_{j}$ in each fluid layer. For the results shown in Section 3 we have set $N_{1,2}=128$. Furthermore, the implemented algorithm has been validated against benchmark problems published in Dongarra, Straughan \& Walker (1996). In addition, we have made extensive comparisons with the results of Li et al. (2007) to verify the effect of the electric field on the stability characteristics. Excellent agreement was found in all cases.

In this section the mathematical method to solve the governing equations for the fluid mechanics and 
the electrostatics has been outlined. The method yields a time-dependent solution for the evolving two-fluid interface in the presence of background shear and electric field effects. In addition, we have described a Chebyshev collocation method for solving the eigenvalue problem that governs the stability of parallel twophase flow. Results of our computational study including comparisons with linear stability analyses will be discussed in the following section.

\subsection{Code Validation}

We begin by considering a simpler problem initially in order to validate the algorithms developed in Section 2. In this model problem a motionless two-phase system of perfect dielectrics is stressed by an electric field. The base state for the electric field is given by the one-dimensional approximation of the Laplace equation (2.14), which reduces to the ordinary differential equation $\left(\epsilon V_{y}\right)_{y}=0$ with Dirichlet boundary conditions on the lower and upper electrode, $V(y=-1)=0$ and $V(y=1)=V_{0}$, respectively. The solution has a jump in the electric field strength across the interface and is accompanied with the development of an electrostatic pressure there. Using the jump condition for the electric displacement $\epsilon_{1} E_{1}(0)=\epsilon_{2} E_{2}(0)$, we find (in dimensional terms)

$$
V_{1}(y)=V_{0} \frac{\epsilon_{2}+\epsilon_{2} y}{\epsilon_{1}+\epsilon_{2}}, \quad V_{2}(y)=V_{0} \frac{\epsilon_{2}+\epsilon_{1} y}{\epsilon_{1}+\epsilon_{2}} .
$$

It follows that the momentum jump condition simplifies to give the electrostatic pressure difference across the interface

$$
\Delta p=p_{1}-p_{2}=\frac{E_{w}}{2}\left[\epsilon_{1} E_{1}^{2}-\epsilon_{2} E_{2}^{2}\right]
$$

Figure 2.3 compares the analytical one-dimensional solutions (2.30) and (2.31) with the numerical approximations computed on three different equidistant grids of $64,128,256$ nodes, respectively, using the front-tracking Navier-Stokes algorithm described in the previous Section. The electric field parameter is $E_{w}=10$ and the ratio of dielectric constants is $\epsilon_{2} / \epsilon_{1}=2$. It is evident that the jump of the electric field across the interface is smeared over some computational cells. By grid refinement the thickness of the transitional zone decreases and the computed profiles collapse onto the analytic solutions. Hence, the results demonstrate the capability of the implemented numerical model to accurately capture electric field effects on the interfacial stress balance in two-layer flow. 

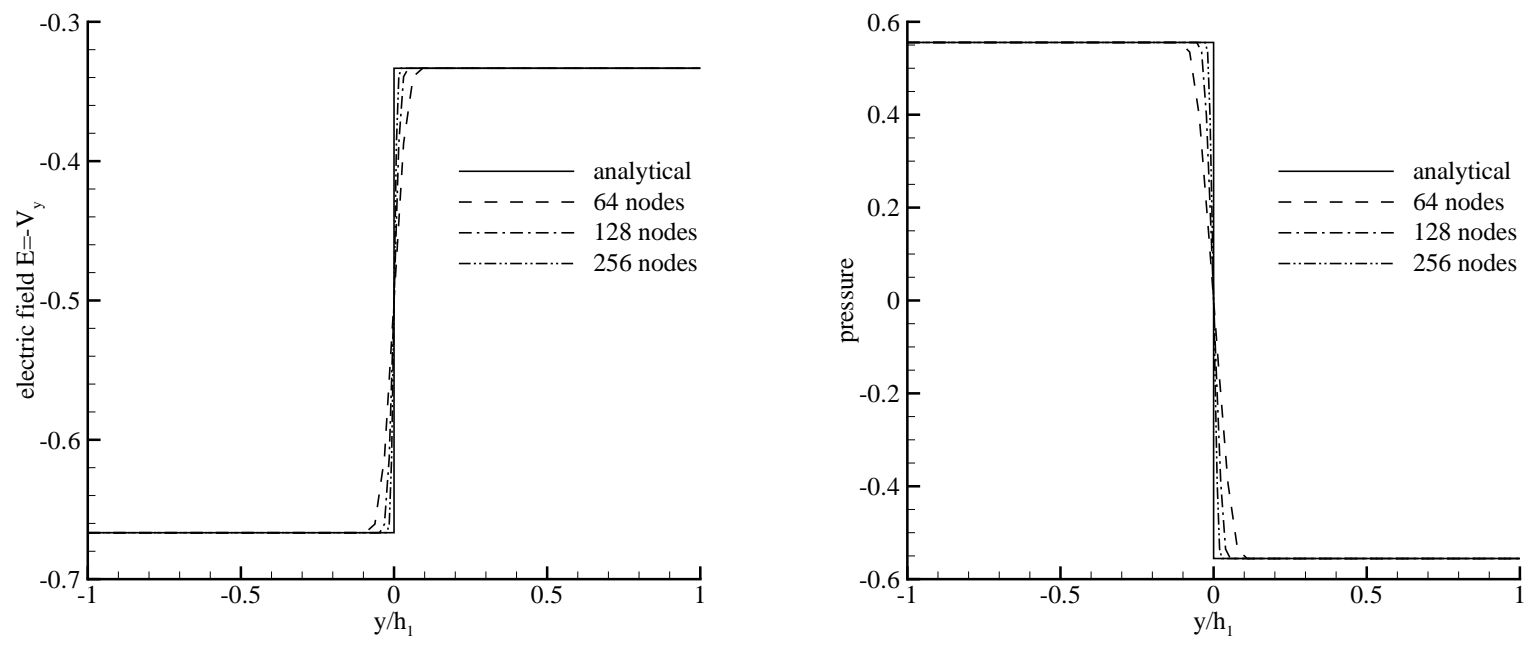

Figure 2. (a) Distribution of the electric field $E=-V_{y}$ computed from equation (2.30) and (b) the pressure given by equation 2.31 for the one-dimensional model problem at $E_{w}=10, \epsilon=2$.

\section{Numerical Experiments}

\subsection{Preliminaries}

With both linear and non-linear computational tools at our disposal, we are in a position to quantitatively evaluate the range of validity of linear theory and provide numerical results that can be useful in the design of operating conditions for different parameters. More importantly our code is capable of following the interfacial evolution into the non-linear regime up to the point it touches one of the bounding walls, an event that is a singularity of the equations of motion since variables such as the pressure, blow up locally. The dimensionless physical parameters governing the flow characteristics are numerous (see expression (2.27), for instance) and in our numerical experiments we restrict this phase space for reasons of computational feasibility. Thus, we fix to unit value the density ratio $\rho$, the undisturbed layer thickness ratio $n$, and the viscosity ratio $m$, and allow parametric variations of the permittivity and conductivity ratios $\epsilon$ and $\sigma$, the Reynolds number $R e$, the surface tension parameter $T$ (this is an inverse Weber number) and the electric field parameter $E_{w}$. Even for this restricted set, we are faced with constructing solution spaces for five parameters (four in the case of perfect dielectrics), a task that is quite challenging and computationally intensive. In order to elucidate the non-linear structures that emerge and also to evaluate linear theory against them, our numerical experiments vary each one of these parameters while holding the others fixed. A complete 
parametric study in the five-dimensional phase-space is implausible computationally and such an effort is of unclear benefit. The applied front-tracking method is capable of handling higher property ratios. In addition to the computations presented next, we have simulated conductivity ratios of 100 or larger and at weak electric field have found no difficulties. However, we can conform that high electrical material property ratios combined with strong electric fields cause numerical instabilities, especially if other material properties (e.g. density and/or viscosity) jump across the interface. We have also tried realistic density and viscosity ratios typically found in air-liquid systems and no evidence of numerical instabilities was found (in the absence of electric fields).

The coupled set of governing equations is solved on a rectangular computational domain with periodic horizontal boundary conditions $(x=0, l)$ and rigid walls at the top $\left(y=h_{2}\right)$ and the bottom $\left(y=-h_{1}\right)$, where we take $h_{1}=h_{2}$ so that the undisturbed interface is at $y=0$. A Couette flow is generated by moving the parallel walls confining the superposed fluids at equal but opposite speeds. Sensitivity studies revealed that a computational grid of $128 \times 128$ nodes is sufficient to accurately capture the physics governing the interfacial dynamics. The transient simulations are started at time $t=0$ from the basic Couette-flow solutions by impulsively perturbing the flat interface with a periodic initial disturbance of the form $s_{i}(x)=s_{0} \cos (x k)$ and zero perturbation velocity everywhere. (Random initial interfacial perturbations of small amplitude were also used and in both cases we find that the dominant linearly unstable mode emerges at large times.) If the flow is unstable, the interfacial perturbations grow in time to non-linear states that eventually impinge onto one of the rigid plates. At such instants the system experiences a touchdown singularity. We note that computational algorithm such as the present one cannot produce accurate solutions in the vicinity of this singular event, nor can it continue the computations beyond this event. Therefore, we terminate the simulations when the minimum distance between a wall and the interface is below 0.005 .

For small initial amplitudes $s_{0}$, we expect the early growth of the perturbations to follow the linear results given by the dispersion relation (2.27) corresponding to the wavenumber $k$ of the imposed initial condition. An initial amplitude $s_{0}=5 \times 10^{-5} h_{1}$ has been chosen (unless stated otherwise) throughout this work and linear growth rates computed using the numerical eigenvalue algorithm described in Section 2.2 are compared with Navier-Stokes simulations which evolve the system into the fully non-linear regime. We found through 
numerical experimentation, that an appropriate and useful comparison can be made most effectively by extracting the exponential growth rate of the interfacial perturbation $s / s_{0}$ versus time where $s$ denotes the maximum amplitude of the interface over the computational domain, by considering log-linear plots in the vicinity of $s / s_{0}=100$, i.e. when the perturbation has had a hundredfold increase in amplitude (such plots are shown in the figures that follow). Note that the interfacial amplitude at this stage is $5 \times 10^{-3}$ and is therefore still small so that linear theory can hold. Note also that such computations enable us to evaluate critical amplitudes above which linear theory becomes inappropriate - as will be seen in the results that follow, EHD Couette flow is described very well by linear theory even for amplitudes having $s / s_{0}=100$. Since the channel has length $l$ and periodic boundary conditions in the horizontal direction are used, only instabilities at wave numbers which represent periodic waves of lengths $l / m$ with $m$ an integer can develop over the computational domain. For this reason, we compare the numerical results with the predicted fastest growing wave, which has a length that matches the periodicity of the problem.

The vertical electric field generates Maxwell stresses that contribute to the stress imbalance at the twofluid interface. For perfect dielectric fluids electric stresses always enhance interfacial motion, while for leaky dielectrics the electric field can either stabilize or destabilize the perturbed interface, depending on the combination of the conductivity and permittivity ratios between the fluids. In contrast to this, surface tension in two dimensions has a purely stabilizing effect and tends to restore the interface to its originally flat state. The system is stable or unstable to linear perturbations about the equilibrium state depending on the ratio of surface tension to electric forces (in terms of our dimensionless groups (2.1) this ratio is equal to $\left.T / E_{w}\right)$. Under unstable conditions, perturbations develop rapidly in time from the imposed initial condition and the evolving interface induces motion in the fluid layers; this motion in turn distorts the electric field due to the re-distribution of the fluid, resulting in a complex non-linear phenomenon. The dominant instabilities are finger-like regions, in which fluid of one layer penetrates into the other, displacing the original fluid sideways. The displaced fluid drains into large hydrodynamically passive regions that form on either side of the penetrating finger. In these reservoirs, incoming momentum diffuses rapidly due to the large amount of accumulated fluid mass. Moreover, viscous stresses and fluid pressure gradients are generated due to the flow 

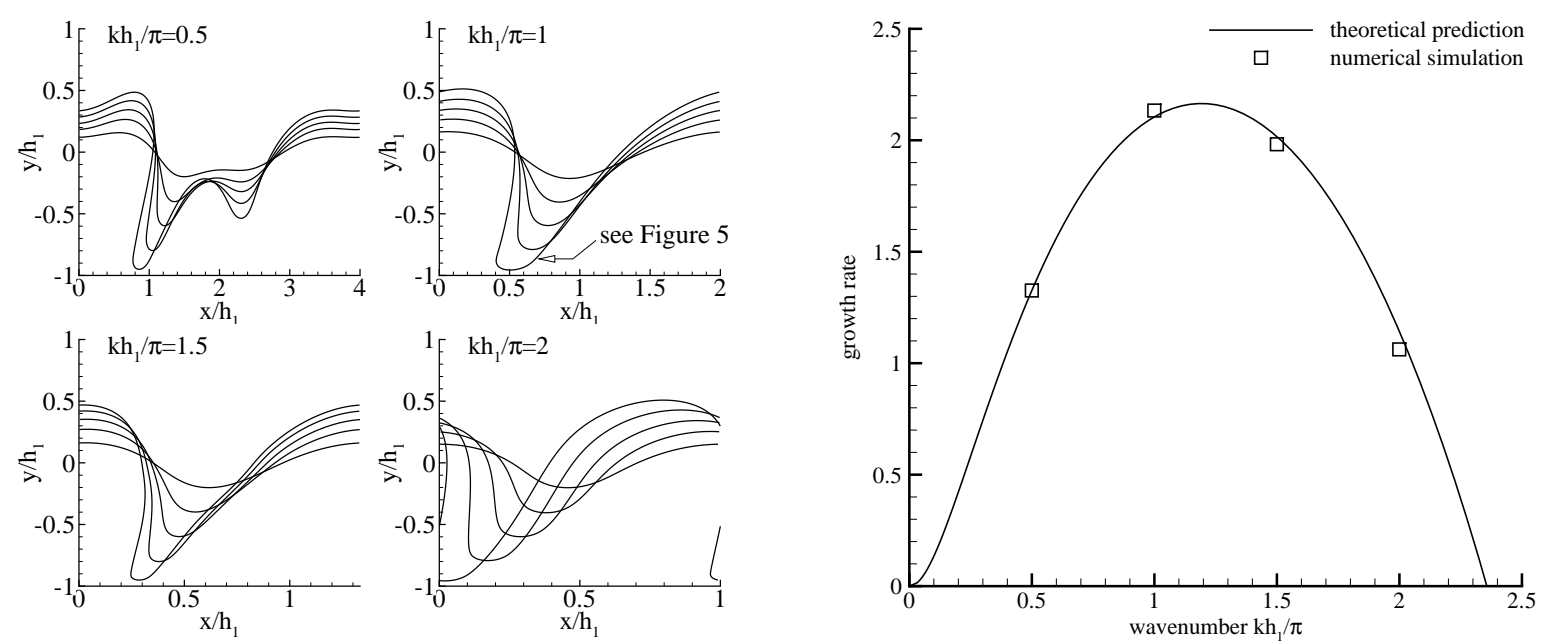

Figure 3. (a) Effect of the wavenumber $k$ of the imposed disturbance on the interfacial instability in plane Couette flow. Temporal evolution of the interface perturbation $s / s_{0}$ and (b) comparison between the numerically simulated growth rates $a$ and the predictions of the linear stability theory $c_{i} k$ for a pair of perfect dielectrics at $R e=10, T=0.5, E_{w}=10, \epsilon=5$.

fields and can also alter the dynamics of the interface substantially. In what follows we describe results for perfect and leaky dielectric fluid pairs.

\subsection{Computations for Perfect Dielectric Fluids}

In this Section we focus on two-fluid systems where the permittivity of the upper fluid layer is higher than that of the lower layer, i.e. $\epsilon>1$. It has been established that a perturbed interface under the action of a vertical electric field deforms towards the fluid with lower permittivity. Physically this is caused by the pressure jump induced by the Maxwell stresses at the interface, which implies that in our simulations the field will tend to distort the interface towards the lower plate electrode; this is indeed the case as shown in the results that follow.

\subsubsection{Effect of perturbation wavenumber - comparison between linear theory and simulations}

We begin by considering the non-linear evolution of initial linear sinusoidal disturbances having increasingly shorter wavelengths characterized by wavenumbers $k h_{1} / \pi=0.5,1,2,4$. For these computations we fix the Reynolds number to $R e=10$, the surface tension parameter to $T=0.5$, the electric parameter $E_{w}=10$ and the permittivity ratio $\epsilon=5$. In figure 3 (a) we show the temporal evolution of the scaled interfacial 
amplitude of $s / s_{0}$ from $t=0$ to times just before the interface touches the wall (the position of the interface is given by $y=s(x, t)$ if it is single-valued, for example, with an arc-length representation for more intricate shapes; in what follows it should be understood that the notation $s / s_{0}$ denotes the interfacial amplitude at a given instant in time). Generally, the deformation of the interface at early times evolves as the sum of exponentially time dependent terms and to identify such growth the interfacial perturbations $s / s_{0}$ are plotted on log-linear scales as mentioned earlier. Using such plots (see figure 3 for example) we have extracted the exponential growth rates for perturbations with wavenumbers given by $k h_{1} / \pi=0.5,1,1.5,2$, over a wide time range and from the vicinity of $s / s_{0}=10^{2}$ as indicated by an arrow in the left panel. Note that in the case of the wavenumbers $k h_{1} / \pi=0.5,2$ the evolution of $s / s_{0}$ departs from its linear behavior just beyond $s / s_{0} \approx 2 \times 10^{2}$, whereas the linear behavior is retained for the perturbation wavenumbers $k h_{1} / \pi=1,1.5$ for much of the duration of the simulation, as can be seen from figure 3. Figure 3 (b) depicts the growth rate $c_{i} k$ predicted by linear stability theory as solid curve. It is found that the interfacial mode is long-wave unstable up to a cutoff wave number $k_{c} h_{1} / \pi \approx 2.36$ and has maximum growth rate $\left(c_{i} k\right)_{\max } \approx 2.16$ at $k h_{1} / \pi \approx 1.2$. Superimposed on this figure are the growth-rate predictions of the direct numerical simulations determined using a least square linear regression fit of the numerical data in the vicinity of the perturbation level $s / s_{0}=10^{2}$, where exponential growth is dominant. We observe a remarkable agreement between the two sets of results and this serves as an additional accuracy check of our numerical work. Additionally, it confirms that linear solutions can be used to describe the flow accurately for amplitudes of $s / s_{0} \approx 10^{2}$. We also carried out a numerical convergence study to determine the behaviour of the computed growth rates on grid resolution. The growth rate was computed for increasingly resolved grids having $64 \times 64,128 \times 128$ and $256 \times 256$ points, respectively. For weakly unstable systems grid refinement has no notable effect on the growth rate, while for highly unstable systems, increases of $1 \%$ at most were observed. In all our tests the slight growth rate increase is monotonic with grid refinement, and a grid of $128 \times 128$ is determined to be sufficiently accurate without a sacrifice in efficiency.

For each of the wavenumber cases depicted in figure 3 we consider next the spatio-temporal evolution of the interface along with the evolution of the maximum pressure on the lower wall of. Results are given in the left and right panels, respectively, of figure 4- all other parameters are the same as in figure 3 , All 

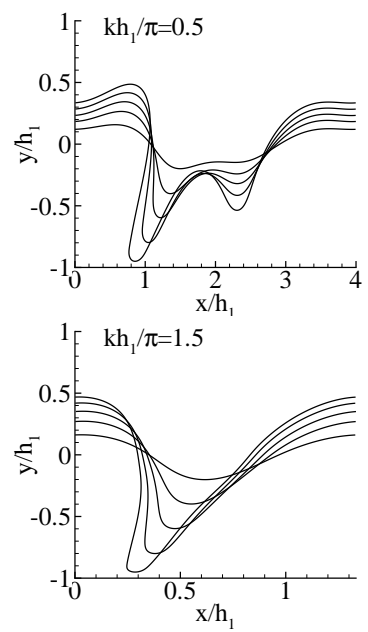
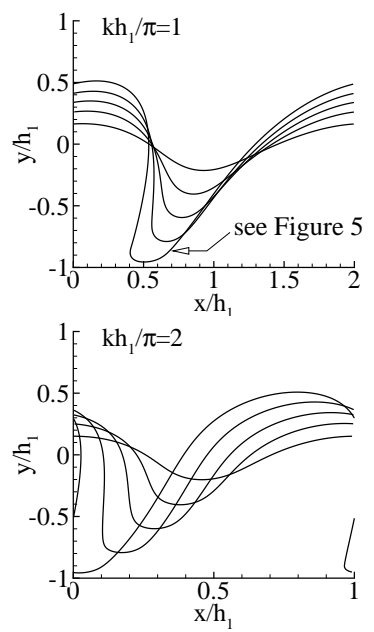

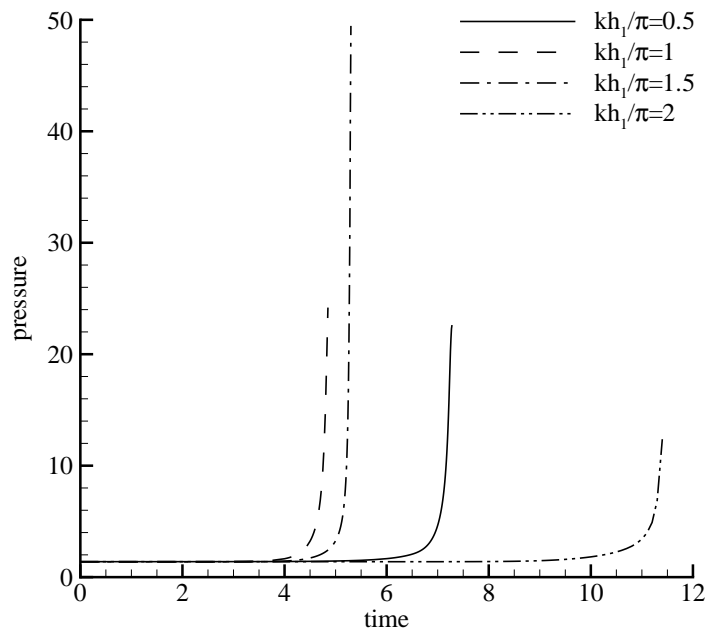

FIGURE 4. Effect of the wave number $k$ of the imposed disturbance on the interfacial instability in plane Couette flow. (a) Temporal evolution of the interface perturbation $s / s_{0}$ and (b) time history of the maximum pressure on the lower plate for a pair of perfect dielectrics at $R e=10, T=0.5, E_{w}=10, \epsilon=5$.

interfacial evolution results (see also figures 8, 10, 12, 14, 17, 20) are constructed by following the initial perturbation into the non-linear regime and plotting snapshots of the interfacial position at times which correspond to the set of successive interface minima $y_{\min } / h_{1}=0.8,0.6,0.4,0.2,0.05$. As a result there are 140 output times that have been recorded for the data presented in these figures and for brevity these are not given here - they are available from the authors upon request. As established above, the interface is linearly unstable with the electric field causing an increase in pressure below an interfacial depression and a decrease in pressure near interfacial elevations. As a result fluid motion ensues from high to low pressure regions, sweeping fluid away from the depression and thus enhancing the instability. As time progresses, the interface develops a fingering instability of interpenetrating fluids from the seeded disturbance (this is a highly non-linear phenomenon). The finger region is initially centered at $x / h_{1}=1$ and moves towards the left as it grows due to the presence of background flow. The case $k h_{1} / \pi=0.5$ is distinct from those having $k h_{1} / \pi=1,1.5,2$, in that it forms multiple fingers before touchdown. The perturbed interface splits into two different branches (in the absence of background flow these branches would possess fore aft symmetry). In all cases the downward elongating finger region induces secondary fluid motion in its neighborhood, which eventually affects the flow field close to the lower plate. Figure 4 (b) depicts the corresponding time histories of the maximum pressure on the lower plate. As discussed above the interface approaches the plate via a 

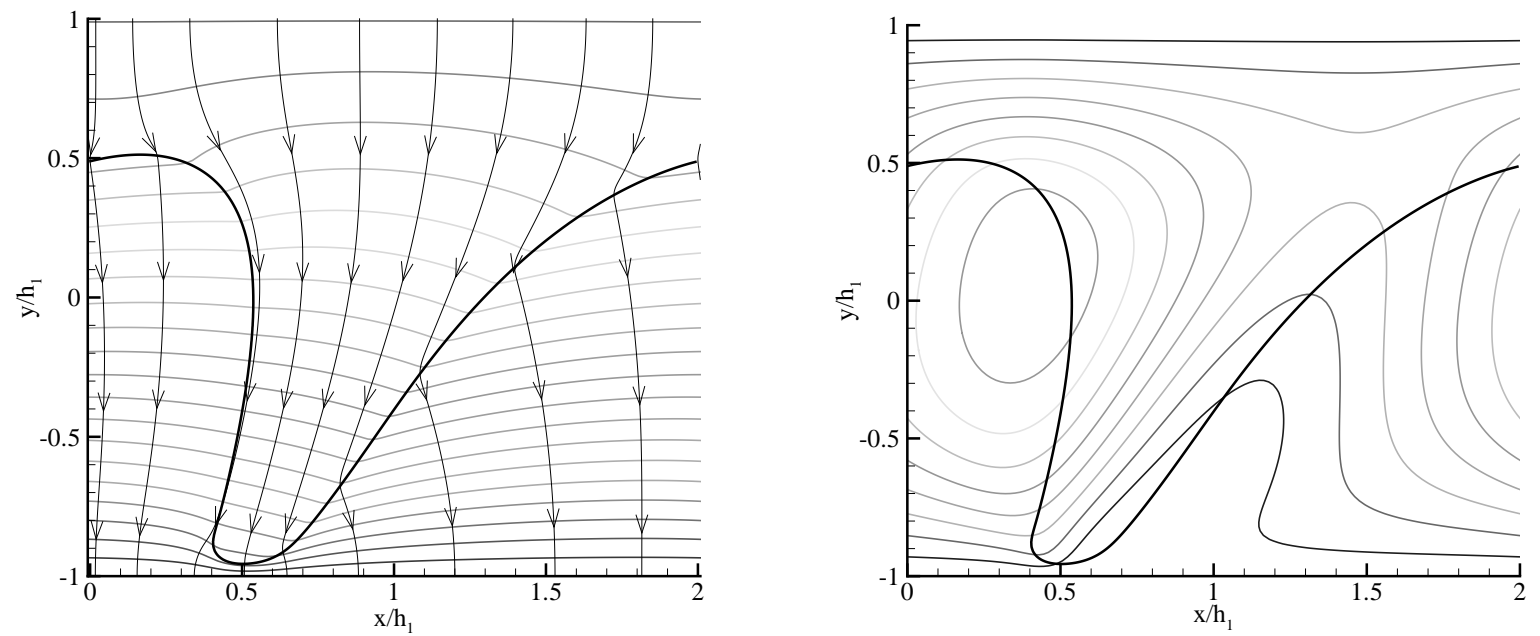

Figure 5. (a) Contours of the electric potential $V$ and electric field lines and (b) the instantaneous streamfunction relative to the background flow for a pair of perfect dielectrics at $l / h_{1}=2, R e=10, T=0.5, E_{w}=10, \epsilon=5$. The time of the snapshot is $t=4.85$. The thick solid line represents the position of perturbed interface.

relatively fast moving finger that terminates in a touchdown singularity. Away from the finger region the system is quasi-static on the time scales of the active finger region. The downward motion of the finger redistributes fluid material in its neighborhood, and eventually affects the pressure distribution on the plate. As seen from the results of figure 4, the pressure retains its initial value as long as the interface is far away from the plate, and then increases rapidly near touchdown terminating in a finite-time singularity. We note that the qualitative nature of the results depends on both the channel height and the initial amplitude of the disturbance.

It is instructive to discuss the structure of the distorted electric field in terms of the voltage potential distribution, noting that in the unperturbed state the equipotential surfaces are parallel to the plate electrodes. In figure 5 (a) we show the equipotential surfaces and corresponding electric field lines at time $t=4.85$, which is close to the touchdown event. The parameters are $l / h_{1}=2, R e=10, T=0.5, E_{w}=10, \epsilon=5$ and this run corresponds to the results of the case $k h_{1} / \pi=1$ of figure 3 . The electric field is depicted with thick lines and the arrows indicate its local direction. Note that the electric potential is strongly perturbed along the interface on the right of the fluid finger, whereas comparatively small distortions are visible on the left. It follows from the electric boundary conditions of the present configuration that the equipotential lines are curved near inclined interface sections but remain mostly straight for vertical and horizontal sections. 

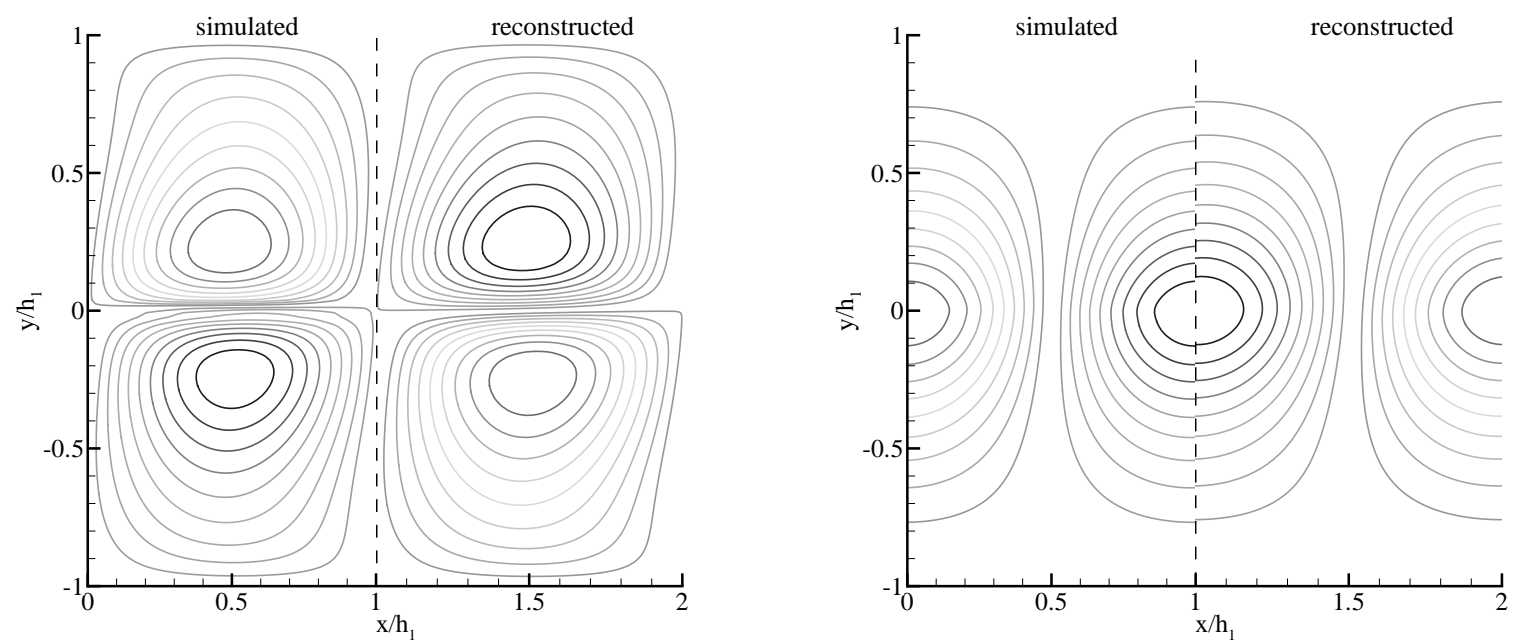

FiguRE 6. Comparison of the simulated velocity perturbations $\tilde{u}(\mathrm{a}), \tilde{v}$ (b) with the corresponding fields reconstructed from the linear analysis for a pair of perfect dielectrics at $l / h_{1}=2, R e=10, T=0.5, E_{w}=10, \epsilon=5$.

According to the definition $\boldsymbol{E}=-\boldsymbol{\nabla} V$ the field lines and the equipotential surfaces form a mutually orthogonal grid. This is evident in our results, as well as the distortion of the electric field due to the presence of fluid regions of different permittivities. The field lines are approximately vertical in the vicinity of the plate electrodes as long as the interface is relatively far away. Close to the interface, however, the slopes of the field lines vary with the local orientation of the interface and differ on either side, explaining why the electric field has its maximum strength in the finger region. In addition to the electric field distribution the instantaneous streamlines at $t=4.85$ are depicted in figure 5 (b) (the streamlines shown represent the perturbed flow the base state has been subtracted). In general, dense and inclined contour lines represent strong secondary flow while widely spaced horizontal or vertical lines indicate hydrodynamically passive flow regions. We can identify relatively low perturbation levels near the upper plate and furthermore in the lateral bulk regions that absorb momentum of the displaced fluid. Along the interface large variations in the streamfunction imply the presence of large perturbation velocities. Note the crest-like patterns to the right and the circular region to the left of the fluid finger, which show the presence of vortical motion.

The linear eigenfunctions of the generalized eigenvalue problem can be utilized to reconstruct the perturbed velocity fields and compare them with the simulations, thus providing additional physical insight into the dynamics of the unstable system at early times. In figure 6 (a) we compare the simulated perturbation field of the horizontal velocity $\tilde{u}$ with the reconstructed field based on the results of the linear stability analysis at 
$k h_{1} / \pi=1, R e=10, T=0.5, E_{w}=10, \epsilon=5$. The numerical data have been evaluated at the perturbation level $s / s_{0}=60$, which corresponds to time $t=2$ in the simulation. The electric field pushes fluid material of the lower layer sideways, which in turn causes a compensating motion of the upper layer towards the center of the interpenetrating region. Moreover, the velocity field is not symmetric due to the presence of background flow. A similar comparison is shown in figure 6 (b) for the perturbed vertical velocity $\tilde{v}$. The traversing interface induces strong downward fluid flow in the finger region accompanied by weak upward motion on the sides of the finger. For both velocity components we observe very good agreement between the simulated and the reconstructed fields, given that the interface has evolved significantly from its initial position. The results of figure 6, therefore, provide additional evidence of the ability of linear theory to predict the early growth of the perturbed system.

\subsubsection{Effect of the electric field parameter $E_{w}$}

The results of Section 3.2.1 were carried out at a fixed strength of the background electric field. Here we compute the flow to touchdown for a range of values of $E_{w}=10,20,30,40,50$, and note that this is an important controlling parameter because it can be changed by simply altering the voltage drop across the device, all other parameters and material properties held fixed. The other parameters in this set of simulations are $l / h_{1}=2$ (the wavenumber of the initial perturbations is $k h_{1} / \pi=1$ ), $R e=10, T=0.5$ and $\epsilon=5$. Results of the evolution of the interfacial perturbation amplitude $s / s_{0}$ along with the theoretically predicted linear growth rates and corresponding numerically simulated ones, are depicted in figure 7 in the left and right panels, respectively. It can be seen that an increase of $E_{w}$ leads to faster growth rates and hence shorter times to wall touchdown - the early time slopes of the curves in figure 7 (a) (where linear theory holds) increase monotonically with $E_{w}$, while the total duration of the computation until the interface comes to within $0.005 h_{1}$ units of the lower wall decreases monotonically. The results in figure 7 (b) compare linear theoretical growth rates with simulated ones estimated as explained earlier from results in the vicinity of $s / s_{0}=10^{2}$ as indicated by the arrow in the left panel, and agreement is once again excellent. Further more there is a conditional instability criterion, namely that the value of $E_{w}$ must be higher than a threshold value of approximately 4 . This can be understood physically by considering the flow characteristics in the 

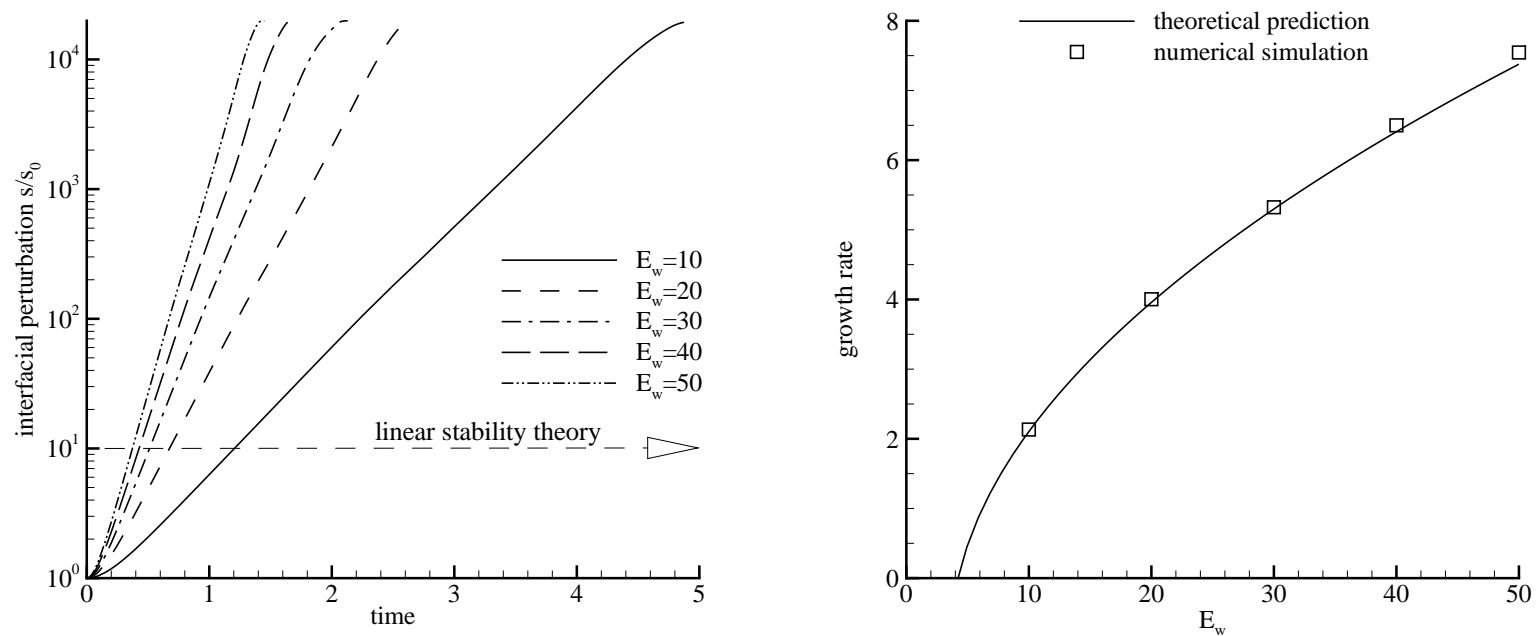

Figure 7. Effect of the electric field parameter $E_{w}$ on the interfacial instability in plane Couette flow. (a) Temporal evolution of the interfacial perturbation $s / s_{0}$ and (b) comparison between the numerically simulated growth rates $a$ and the predictions of the linear stability theory $c_{i} k$ for a pair of perfect dielectrics at $l / h_{1}=2, R e=10, T=0.5, \epsilon=5$.

absence of a field where the classical two-phase Couette problem holds. In our simulations the viscosity ratio is $m=1$ which makes the interfacial mode stable at all Reynolds numbers including $R e=10$ used in these results. A small electric field, therefore, is incapable of overcoming interfacial damping, but can make the interface unstable if it is sufficiently large, in complete agreement with the results of figure 7

Next we consider the interfacial evolution to touchdown at the increasing sequence of values of $E_{w}=$ 20,30,40,50 (for comparison, analogous results for $E_{w}=10$ can be found in figure 4 ); the results are given in figure 8 (a). For the two weakest values $E_{w}=10,20$ a single finger of the penetrating upper fluid develops with touchdown taking place at a single point. Increasing the field strength causes the initial perturbation to spread laterally, leading to multiple fingers as can be seen from the results. For $E_{w}=30$, for example, two fingers are formed and are driven away from the center-plane $x / h_{1}=1$ leading to an almost simultaneous touchdown at the two approximate positions $x / h_{1} \approx 0.25$ and $x / h_{1} \approx 1.25$; this in turn leads to the formation of a smaller drop of the lower fluid, a non-linear phenomenon reminiscent of satellite drop formation in liquid jet breakup. (We have verified numerically - not shown - that the number of fingers forming as $E_{w}$ increases, is due to nonlinear effects because the maximally unstable wavelength is of the order of the computational domain and is rather insensitive to the electric field strength for $E_{w}$ larger than about $10-20$ and less than 50, approximately; at the same time the cut-off wavelength decreases with increasing $E_{w}$ as expected.) As 

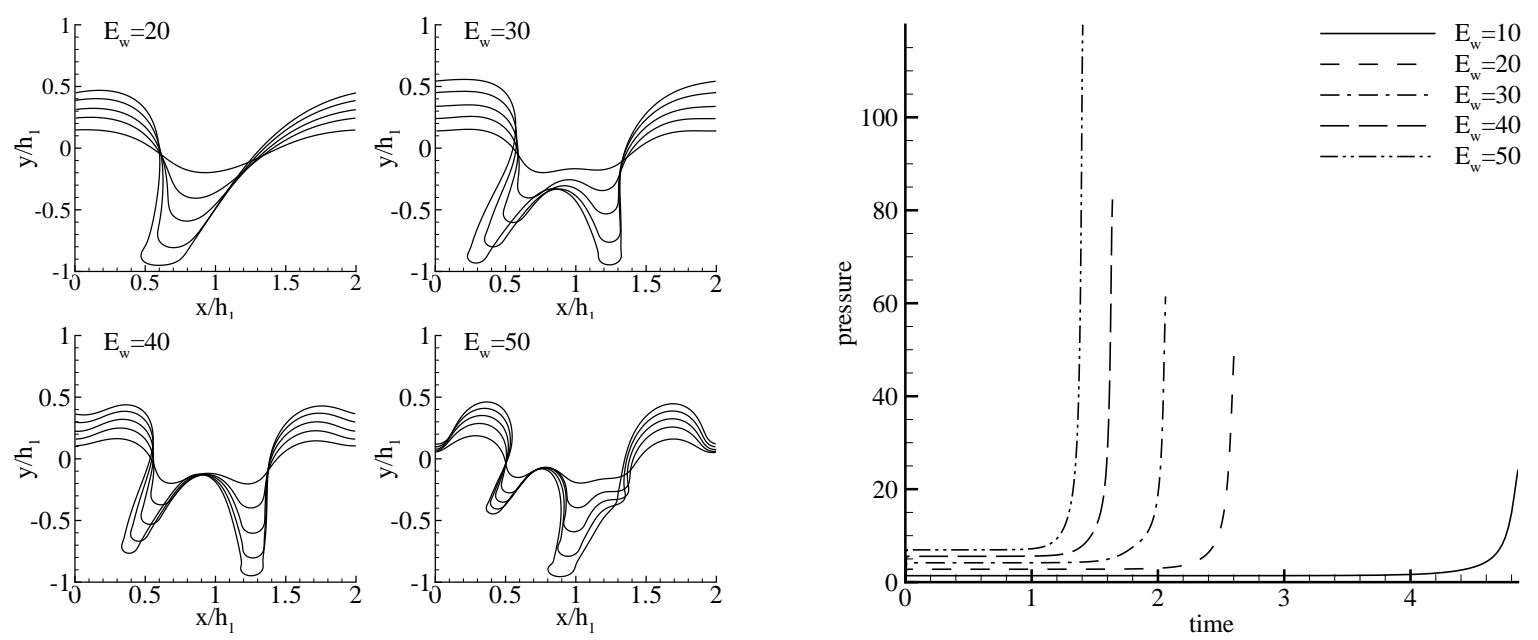

Figure 8. Effect of the electric field parameter $E_{w}$ on the interfacial instability in plane Couette flow. (a) Temporal evolution of the perturbed interface shape and (b) time history of the maximum pressure on the lower wall for a pair of perfect dielectrics at $l / h_{1}=2, R e=10, T=0.5, \epsilon=5$.

$E_{w}$ is increased further to a value of 40 , two fingers emerge again but the right one grows significantly faster than the left one and touches down first at a position $x / h_{1} \approx 1.25$. This asymmetric growth originates from the presence of the background flow and is amplified by the electric field. Even though our computations must stop at touchdown, we anticipate that the left finger will continue its trajectory to touchdown and will not be affected significantly by the topological transition and contact line problem ahead, thus producing a satellite as predicted in the case of $E_{w}=30$. The tendency of the interface to develop multiple fluid branches is even more visible in the case $E_{w}=50$, where the left branch becomes stunted to a dimple on the interface, while the faster growing right branch clearly dominates the structure of the evolving interface. In addition to these two fingers, a third branch emerges on the right side of the fastest growing finger. The touchdown position is also affected and occurs to the left of the mid-plane $x / h_{1}=1$. Furthermore, we note in passing that higher values of $E_{w}$ lead to the formation of an even more ramified interface with multiple fingers appearing.

As the interface evolves to touchdown on the lower plate, the pressure distribution at the wall appears to be going singular in finite time, a phenomenon found earlier for different parameters. The evolution of the maximum lower wall pressure is depicted in the results of figure 8 (b), and it is seen that the time to touchdown decreases non-linearly as $E_{w}$ is increased. For example, doubling $E_{w}$ from 10 to 20 results in a 

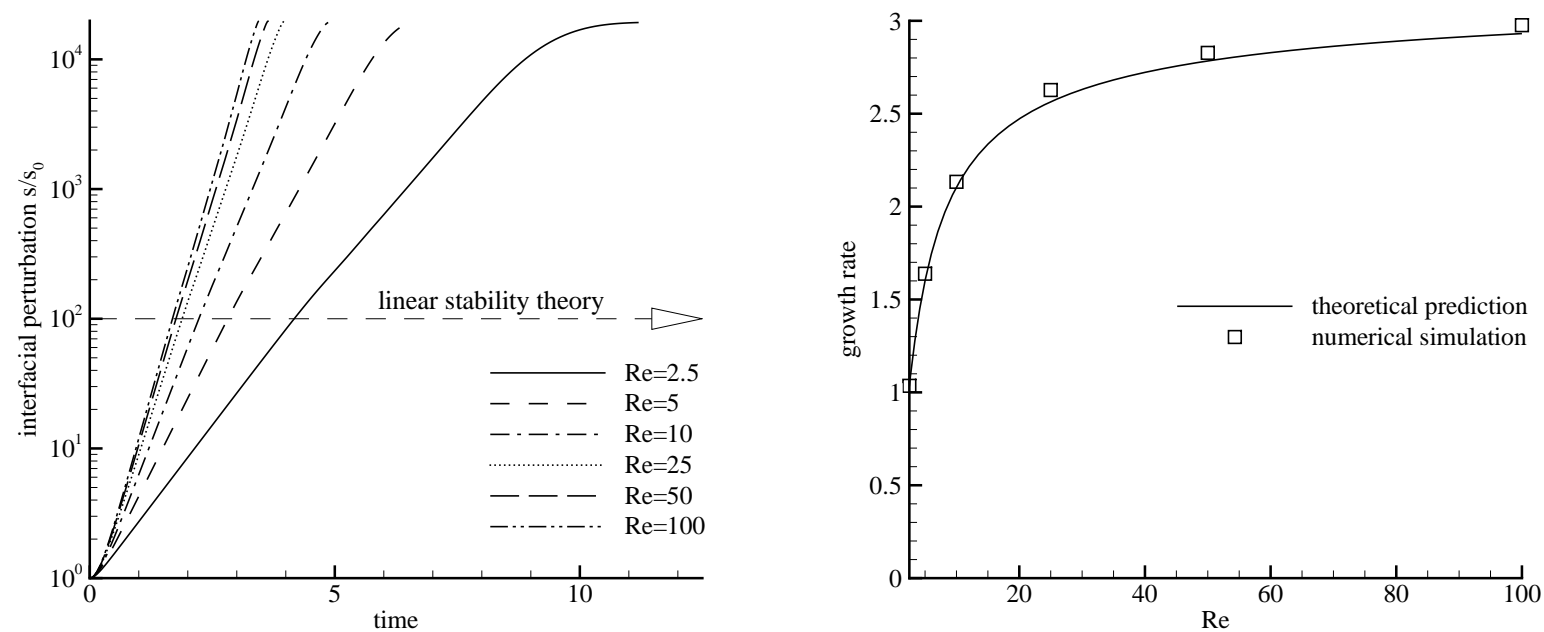

FIGURE 9. Effect of the Reynolds number Re on the interfacial instability in plane Couette flow. (a) Temporal evolution of the interfacial perturbation $s / s_{0}$ and (b) comparison between the numerically simulated growth rates $a$ and the predictions of the linear stability theory $c_{i} k$ for a pair of perfect dielectrics at $l / h_{1}=2, T=0.5, E_{w}=10, \epsilon=5$.

decrease of the touchdown time from approximately 4.8 to 2.6 , whereas a further doubling of $E_{w}$ to a value of 40 results in touchdown after $t \approx 1.6$. These results are in line with the linear growth rate predictions presented in figure [7. Recalling that the computations are stopped when the interface evolves to a fixed small distance from the wall (this distance is $0.005 h_{1}$ ), we can also explain the fact that an increase in the value of $E_{w}$ is accompanied by an increase in the value of maximum pressure attained when the interface reaches a fixed distance from the wall.

\subsubsection{Effect of the Reynolds number Re}

In addition to the electric stresses that drive the instabilities described above in perfect dielectric two-layer systems, inertial effects also play a significant role in the stress balance and instability characteristics. In what follows we consider the effect of the Reynolds number $R e$ on the initial and long-time evolution of the flow, in the presence of an electric field and all other parameters held fixed. In the numerical experiments reported next we fix $l / h_{1}=2, T=0.5, E_{w}=10$ and $\epsilon=5$, so that the flow is unstable (at $R e=10$ at least) as discovered from the results of figure 7 As already mentioned, our study focuses on viscosity-matched fluids where viscous effects appear as a momentum diffusion mechanism which stabilizes the interface in the low Reynolds number regime where inertial effects are small. In figure 9 (a) we show the evolution to touchdown 

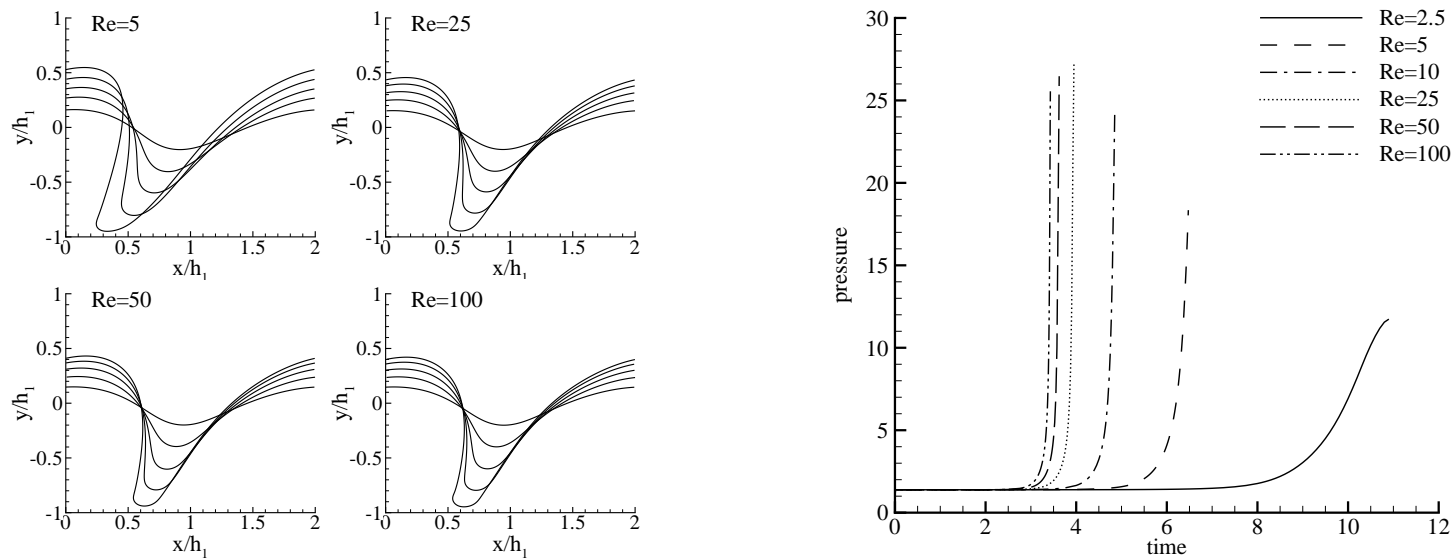

Figure 10. Effect of the Reynolds number Re on the interfacial instability in plane Couette flow. (a) Temporal evolution of the perturbed interface shape and (b) time history of the maximum pressure on the lower plate for a pair of perfect dielectric fluids at $l / h_{1}=2, T=0.5, E_{w}=10, \epsilon=5$.

of the maximum interfacial perturbation $s / s_{0}$ for the set of Reynolds numbers $R e=2.5,5,10,25,50,100$, and it is seen that exponential growth associated with linear theory is driving the system at early times as expected. The growth rates increase monotonically with $R e$ as seen from the results of figure 9 (b) which compares theoretically predicted linear values with numerically simulated growth rates for the chosen initial condition; agreement is once again excellent. Interestingly, the growth of the interfacial perturbation at $R e=2.5$ shows a remarkable decrease, with $s / s_{0}$ flattening out as the interface approaches the plate. Since the growth rate at this Reynolds number is relatively small (see figure 9 (b)) a secondary flow field forms around the wider region of the evolving interface and the rigid plate affects the evolution at earlier stages. As a result the growth rate evidently drops at the final stages when the interface comes very close to the lower plate. With an increase in Reynolds number the interfacial perturbations develop at higher rates and due to the fast growth of the finger the secondary flow shrinks to a narrow region around the evolving interface. This explains why at higher Reynolds numbers the presence of the plate affects the interfacial dynamics at smaller time intervals from the touch-down event.

For completeness we present the non-linear evolution of the interface to touchdown for the values $R e=$ $2.5,5,25,50,100$ (for comparison with analogous results at $R e=10$ we refer to figure 4 ). The results are given in figure 10 (a). It is evident that a single finger of interpenetrating fluid forms for this range of Reynolds numbers. For small Reynolds numbers, i.e. $R e=5$, the growth rates of the perturbed interface are relatively 

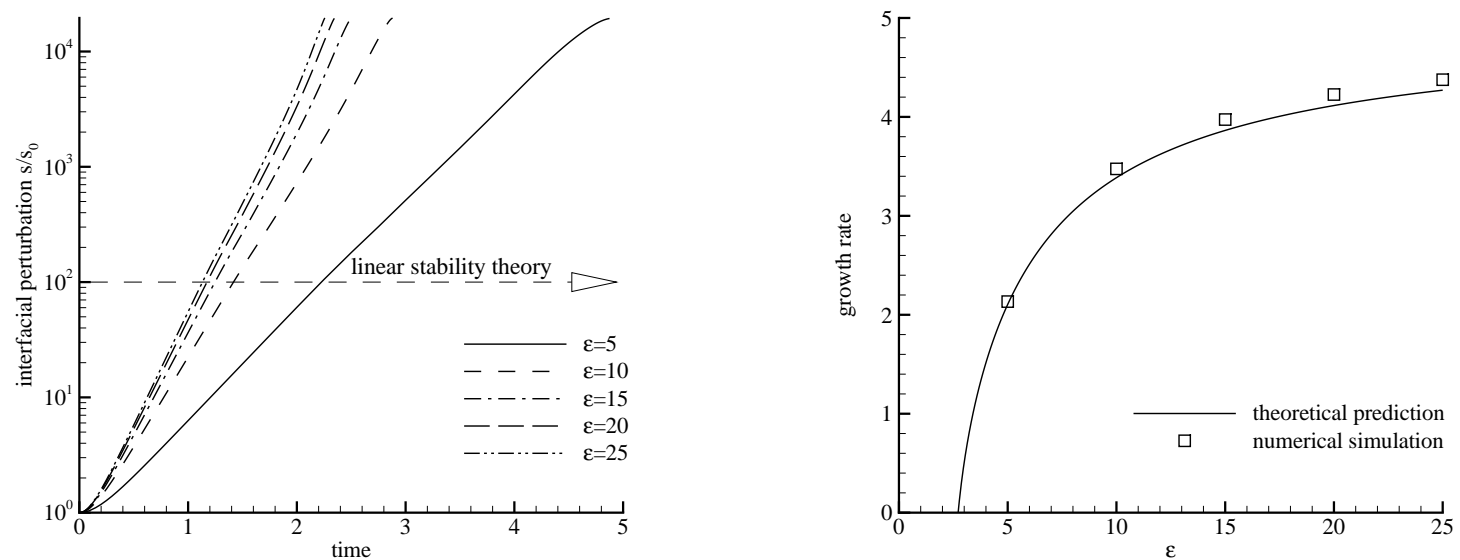

Figure 11. Effect of the permittivity ratio $\epsilon$ on the interfacial instability in plane Couette flow. (a) Temporal evolution of the interface perturbation $s / s_{0}$ and (b) comparison of the numerically simulated growth rates $a$ and the prediction of the linear stability theory $c_{i} k$ at $l / h_{1}=2, R e=10, T=0.5, E_{w}=10$.

small and the local background Couette flow effectively propagates the slowly evolving finger towards the left. Increasing the Reynolds number leads to higher growth rates (see figure 9), producing faster elongating fingers and altering non-linearly the horizontal base flow and the interplay between interfacial dynamics and background flow. Moreover, inertia becomes locally important as the tip velocities increase, and as a result a tapered finger forms with increasing Reynolds number since fluid in the neck region cannot follow the fast growing finger tip. At the same time, the flow around the finger tip causes a rapid increase of the pressure on the lower plate immediately before the touch-down event occurs. Figure 10 (b) shows the corresponding time history of the maximum pressure on the lower plate and it can be concluded that the pressure seems to encounter a singularity in finite time for all cases except when $R e=2.5$ where the numerical results indicate that it reaches a local maximum just before the computation is stopped at a prescribed minimum distance from the wall. This particular case will be investigated in future work including additional analysis in the Stokes flow regime.

\subsubsection{Effect of the permittivity ratio $\epsilon=\epsilon_{2} / \epsilon_{1}$}

The physical effect of increasing the permittivity ratio is to produce stronger Maxwell stresses, enhance the stress imbalance at the two-fluid interface and directly affect the stability of the system. Figure 11(a) shows the temporal evolution to the interfacial perturbation $s / s_{0}$ for the permittivity ratios $\epsilon=5,10,15,20,25$. 
The other parameters are fixed to $l / h_{1}=2, R e=10, T=0.5, E_{w}=10$. Figure 11 (b) depicts the growth rate as a function of $\epsilon$ and the comparison between linear theory and simulation is once again excellent (the comparison for this run is made in the vicinity of $s / s_{0}=100$ as indicated on the figure). The critical value of $\epsilon$ below which the flow is stable in the results of figure 11, is the result of the competition between the stabilizing effect of surface tension and the vertical electric field, which is inherently destabilizing for perfect dielectric systems. Reducing the surface tension causes a more unstable system, where the critical instability threshold is shifted towards smaller permittivity ratios, as confirmed here from both linear theory and direct simulations of the Navier-Stokes equations. There are several notable features in these results. First, the evolution to touchdown becomes monotonically faster as $\epsilon$ increases and this can be seen clearly from the results in the left panel. Regarding linear theory and corresponding simulations (figure11(b)), such monotonic increase corresponds to the monotonically increasing growth rates presented in the figure. As $\epsilon$ becomes larger (e.g. approximately 15 and higher), the growth rate levels off and the curves $s / s_{0}$ become closer to each other as seen in figure11(a). The physical explanation for this is that for $\epsilon \gg 1$ the lower fluid behaves as a perfect conductor, to leading order, with the interface becoming an equipotential surface. It has been established that such flows are unstable if the electric field is strong enough (see Craster \& Matar 2005), as is the case for the present set of parameters. The non-linear dynamics also tend asymptotically to those of the perfectly conducting case and this has been computed and analyzed by Tseluiko et al. (2008) in a related problem of falling films over topographically structured substrates.

Spatial details of the evolving interface and the corresponding maximum pressure time histories, are given in figure 12 (a) and (b), respectively, with the values of $\epsilon$ labeled on the figure. At low values of $\epsilon$ (e.g. $\epsilon=10)$ continuously tapering fingers of the interpenetrating fluid emerge from the perturbed interface, while at larger values of $\epsilon$ drop-like tips connected with long thinning fluid necks are found. In addition to the drop-neck formation computed at large $\epsilon$, a bifurcation takes place with additional fingers emerging; this phenomenon is already evident at $E_{w}=20,25$ and becomes much more prominent at even higher permittivity ratios (not shown). Considering the evolution of the maximum lower-wall pressure, we observe that its final computed value (at a fixed equal distance from the wall) monotonically increases with the 
Interfacial Instability in Electrified Plane Couette Flow
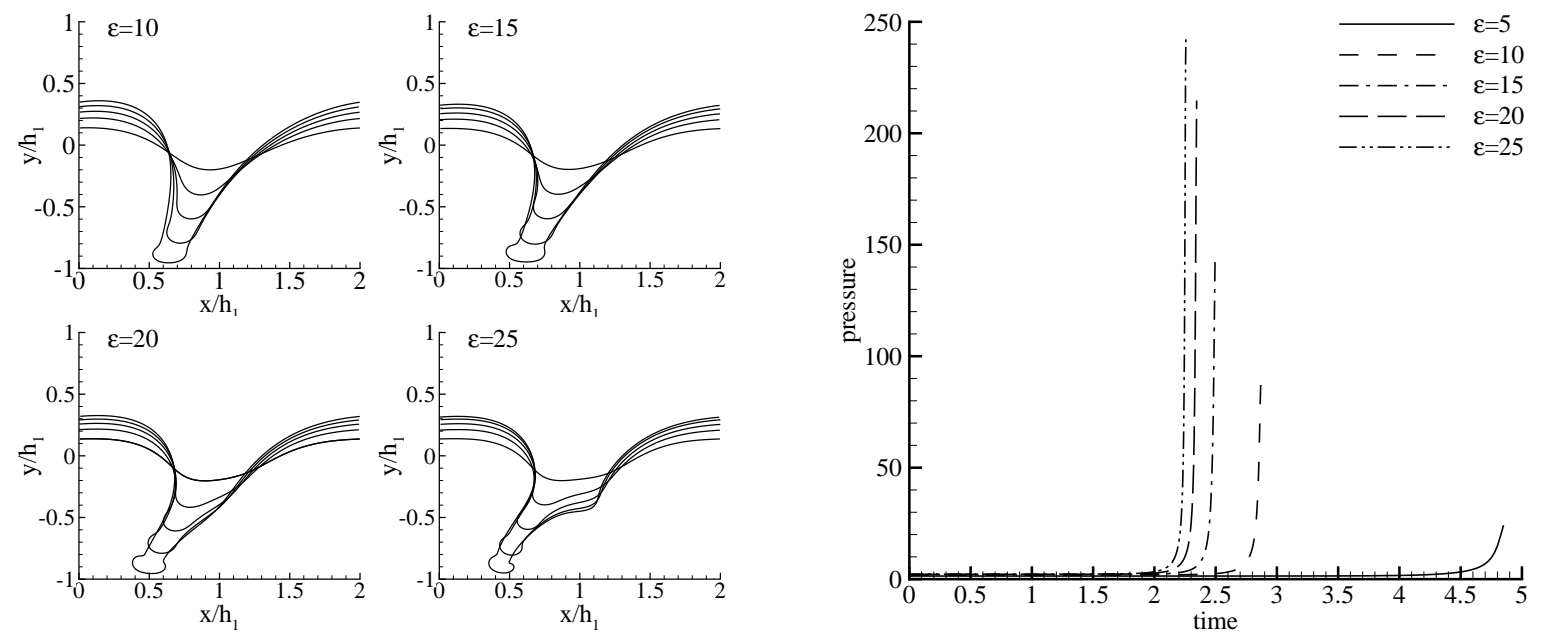

FIGURE 12. Effect of the permittivity ratio $\epsilon$ on the interfacial instability in plane Couette flow. (a) Temporal evolution of the interfacial perturbation $s / s_{0}$ and (b) comparison between the numerically simulated growth rates $a$ and the prediction of the linear stability theory $c_{i} k$ at $R e=10, T=0.5, E_{w}=10$.
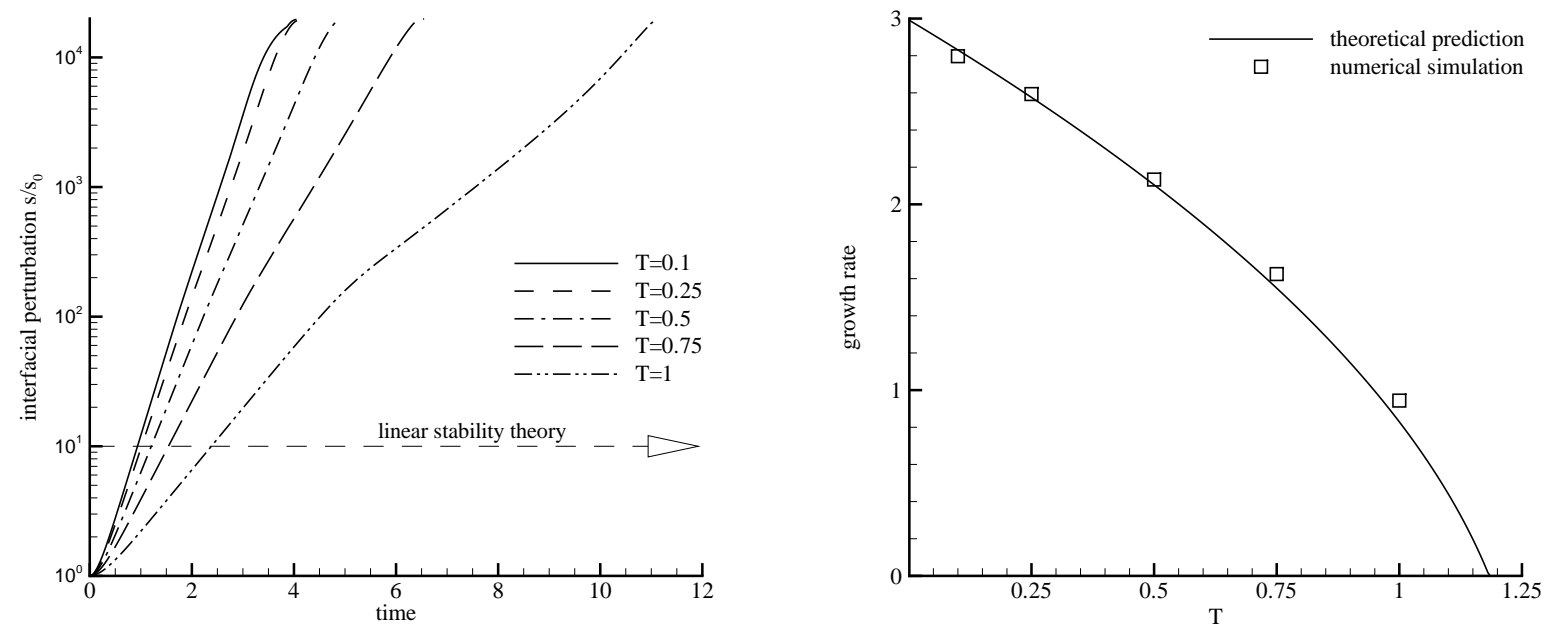

FIGURE 13. Effect of the surface tension parameter $T$ on the interfacial instability in plane two-layer Couette flow. (a) Temporal evolution of the interfacial perturbation $s / s_{0}$ and (b) comparison between the numerically simulated growth rates $a$ and the predictions of the linear stability theory $c_{i} k$ for a pair for perfect dielectrics at $l / h_{1}=2, R e=10, E_{w}=10, \epsilon=5$.

permittivity ratio. In addition, the time to touchdown decreases monotonically as $\epsilon$ increases and this is also supported by the growth rate calculations presented earlier. 


\subsubsection{Effect of the surface tension parameter $T$}

We complete our parametric study of leaky dielectric fluids pairs by considering variations in $T$, all other parameters fixed at $l / h_{1}=2, R e=10, E_{w}=10, \epsilon=5$. Note that for $T=0.5$ the flow is unstable as described in previous sections. The prominent physics of surface tension effects in two dimensional interfacial flows are stabilization of short waves (either damping or dispersive) due to the large size of the curvature for short-wave features. A cutoff wavenumber $k_{T}$ exists, therefore, above which the flow is linearly stable, and $k_{T}$ decreases as the $T$ increases. In this section we extend such linear notions into the non-linear regime by simulating the flow and results are presented in figure 13. The left panel shows the evolution of $s / s_{0}$ and the results confirm linear exponential growth for all values of $T$ at early times. As expected, the linear growth rate decreases with increasing values of $T$ and for the lower values (e.g. $T=0.1,0.25,0.5)$ the results indicate that linear theory is a very good predictor until times close to touchdown where non-linearity must enter. It is interesting to note that at the highest surface tension value considered $(T=1)$, the growth rate attenuates after the perturbation has reached a level $s / s_{0} \approx 2 \times 10^{2}$ and eventually increases again, a phenomenon that is clearly non-linear. Figure 13 (b) compares the numerically simulated growth rates with the linear stability results. Linear theory for the given system predicts a stable regime for $T>1.19$, and the growth rates estimated from the simulation are once again in excellent agreement with theory (the comparison is made at the value $s / s_{0}=10^{2}$ as indicated in the figure). Physically, the existence of a critical value of the surface tension parameter $T$ above which the flow is stable, stems from the stabilising effect of surface tension on two-dimensional interfacial disturbances.

The interfacial evolution is depicted in figure 14 (a) for the set $T=0.1,0.25,0.75 .1 .0$. At the smallest value of $T=0.1$ there exist many unstable modes and the interface experiences a rich variety of nonlinear instability patterns; the initial single-valued perturbation splits into two interpenetrating fingers at relatively early times. Due to the presence of background flow these fingers grow at different rates and different orientations with respect to the vertical. As a result, a downwards moving, strongly elongated branch forms on the left, while the branch on the right becomes stunted as it propagates upstream with respect to the direction of the local background flow. We have found similar phenomena at $T=0.2$ (not shown) and the complexity of these fingers increases in the limit $T \rightarrow 0$. In contrast, at larger values of $T(T=0.25,0.75,1.0$ 

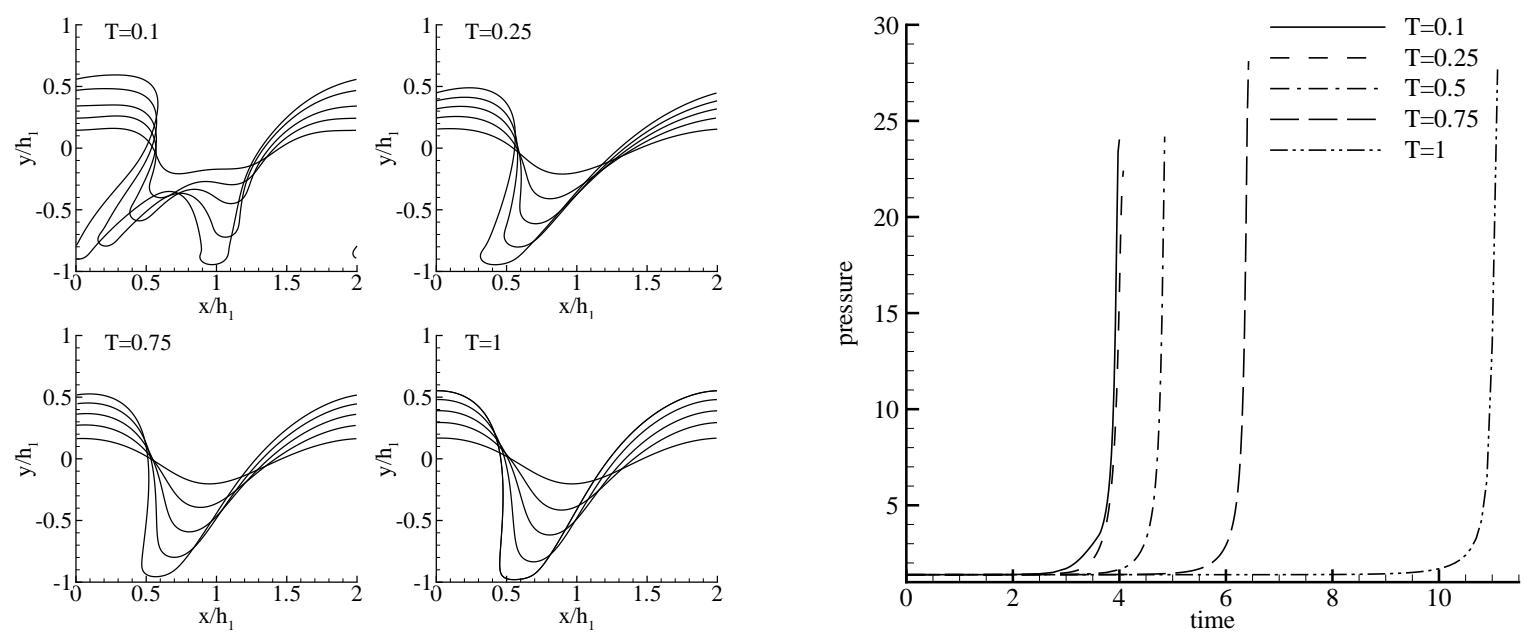

FIGURE 14. Effect of the surface tension parameter $T$ on the interfacial instability in plane two-layer Couette flow. (a) Temporal evolution of the interfacial perturbation $s / s_{0}$ and (b) comparison between the numerically simulated growth rates $a$ and the predictions of the linear stability theory $c_{i} k$ for a pair of perfect dielectrics at $l / h_{1}=2, R e=10, E_{w}=10, \epsilon=5$.

and figure 4 for $T=0.5)$ single fingers form due to surface tension stabilization and the emergence of a long-wave regime. Moreover, the interpenetrating finger widens as the regularizing effects of surface tension increase with increasing values of $T$. A change in slope, that delays touchdown, can be observed in the evolution of the interfacial perturbation (figure 14, (a)) for the two largest values of $T=0.75,1.0$, during the nonlinear stages of the dynamics. The physical mechanism responsible for this behaviour is the stabilising influence of surface tension which is promoted at these values of $T$ and competes with the destabilising electrohydrodynamic forces to produce the observed nonlinear phenomena. We emphasize that the size and width of the finger depends primary on the competing effects of surface tension, which tends to restore the original flat interface, and electric stresses, which tend to produce elongated structures. We complete this case by discussing the effect of surface tension on the time history of the maximum pressure on the lower-plate (figure 14 (b)). The interfacial touchdown is eventually accompanied by a singularity in local pressure and as expected the time to the singularity increases. The vigorous stabilization of surface tension is evidenced by the doubling (approximately) of the time to touchdown by a $30 \%$ increase in the value of $T$ from 0.75 to 1.0 . 

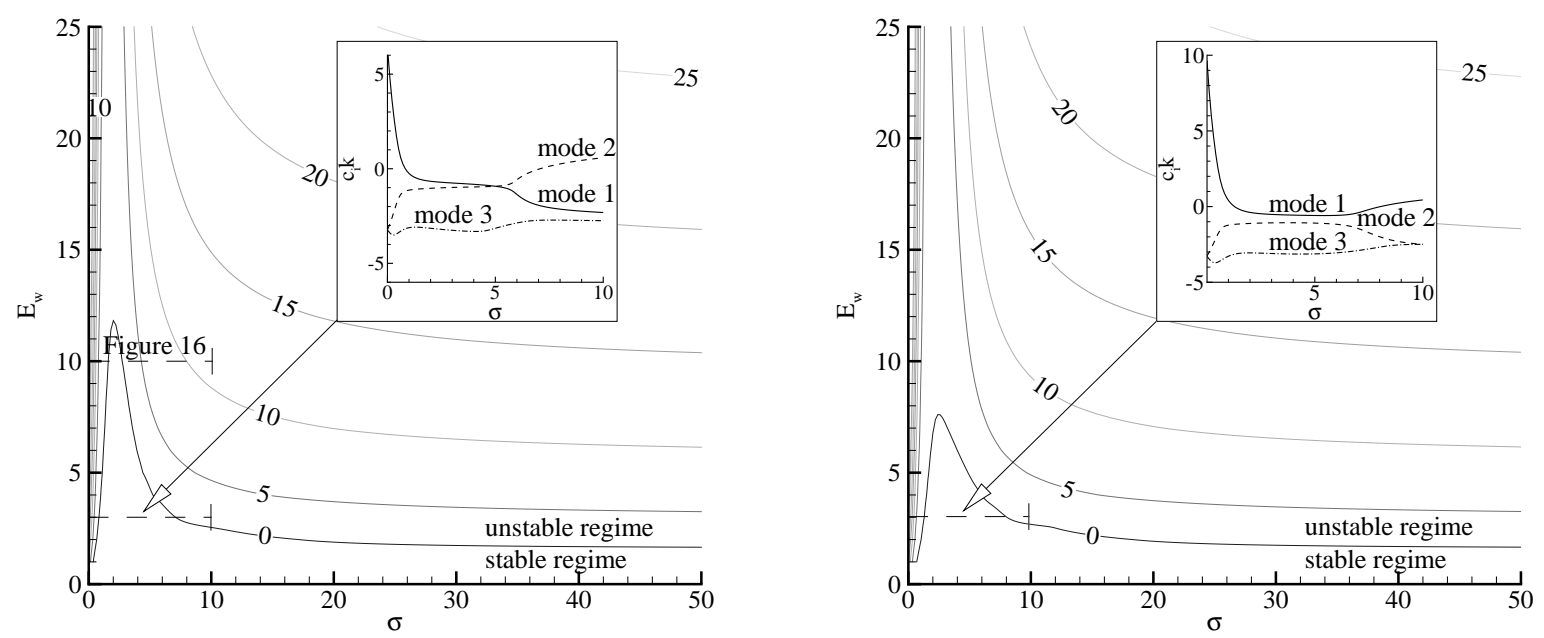

Figure 15. Effect of the conductivity ratio $\sigma$ on the interfacial instability in plane two-layer Couette flow. Lines of constant growth $\left(c_{i} k=\right.$ const.) for $(\mathrm{a}) \epsilon=5$ and $(\mathrm{b}) \epsilon=10$ for a pair of leaky dielectric fluids at $l / h_{1}=2, R e=10, T=0.5$.

\subsection{Computations for Leaky Dielectric Fluids}

So far the we focussed on the EHD instability of interfaces separating perfect dielectric fluids and established that a vertical electric field is destabilizing and causes touchdown of the interface with the lower wall in finite time (the lower fluid has the smaller permittivity in all our computations). Even a small amount of conductivity, however, enables charges to reach the interface, form a diffuse charge layer there and modify both the normal and tangential stress balances; the latter modification is absent in both perfect conductors and perfect dielectrics and hence the model allows for additional physical effects. In addition to $R e, \epsilon, T$ and $E_{w}$, the parameter $\sigma=\sigma_{2} / \sigma_{1}$ enters which measures the ratio of upper to lower fluid conductivities. The combined effect of $\sigma$ and $\epsilon$ creates a rich variety of interfacial instabilities in leaky dielectrics and physically an additional coupling with the hydrodynamics enters through the tangential stress balance which is now modified due to the Maxwell stresses. Our objective is to quantify such effects into the non-linear regime using direct simulations along with comparisons with linear theory, where meaningful.

\subsubsection{Effect of conductivity ratio $\sigma$}

The generalized eigenvalue problem described in Section 2.2 is solved and results are presented by constructing stability diagrams as the five parameters $R e, \epsilon, \sigma, T$ and $E_{w}$ vary. Figure 15 depicts lines of constant 
growth $\left(c_{i} k=\right.$ const.) labeled on the diagram, in the $\sigma-E_{w}$ space, for a pair of leaky dielectric fluids in a channel with geometry $l / h_{1}=2$ and fixed values of $R e=10, T=0.5$. Figure 15 (a) has permittivity ratio $\epsilon=5$ while Figure 15 (b) contains results at the higher value $\epsilon=10$. By investigating the linear eigenvalue spectrum $\left(c_{r}, c_{i}\right)$ we found that at large field strengths the leaky dielectric system possesses one unstable mode, while all other modes in the discrete spectrum are stable. By contrast, we identified two distinct unstable modes at low conductivity ratios and weak electric fields (see the inset of figure 15 which shows results representing the growth rate at fixed $E_{w}=3$ along the dashed line $0 \leq \sigma \leq 10$ indicated on the figure). More specifically, a highly unstable mode (mode 1 ) emerges at $\sigma=0$ and becomes rapidly stabilized as $\sigma$ increases. In turn, a second mode (mode 2) that is stable for small $\sigma$, loses stability and eventually becomes the dominant unstable mode at sufficiently large values of $\sigma$ (above 6 approximately). Near the crossing point these two modes have nearly identical phase velocities $c_{r}$. A third mode (mode 3 ) is also depicted but this is stable over the whole range of $\sigma$ considered.

Increasing the permittivity ratio to $\epsilon=10$ does not modify the qualitative stability characteristics as illustrated in figure 15 (b). Overall, the lines of constant growth rate have similar characteristics with those of $\epsilon=5$, with the exception of the low conductivity ratio and weak electric field regimes. For the particular set of parameters used in these calculations, the stable regime shrinks to smaller values of $E_{w}$, hence instability is enhanced. It is also interesting to note that there is no mode crossing of unstable modes for $E_{w}=3$, in contrast to the lower permittivity case $\epsilon=5$, implying that there is only one mode in the eigenvalue spectrum which becomes unstable in this case. As seen in the details of the inset in figure 15 (b), at small conductivity ratios the dominant mode is the same as that found at the lower permittivity ratio $\epsilon=5$ depicted in figure 15 (a). We note that similar conditional stability results were found by Posner \& Santiago (2006) in a different context involving miscible electrolyte solutions, and the effect was attributed to an electric Rayleigh number with diffusion being important. In the present immiscible system the conditional stability criteria result from a competition between normal and tangential electrical stresses, the latter being supported in immiscible leaky dielectric systems (see Li et al. 2007 for an extensive discussion of such linear stability properties).

Guided by the linear stability results of figure 15 we compute the flow into the non-linear regime for different $\sigma$ along the line $E_{w}=10$ as indicated in figure 15 (a) (i.e. $R e=10, \epsilon=5, T=0.5, E_{w}=10$ ). 

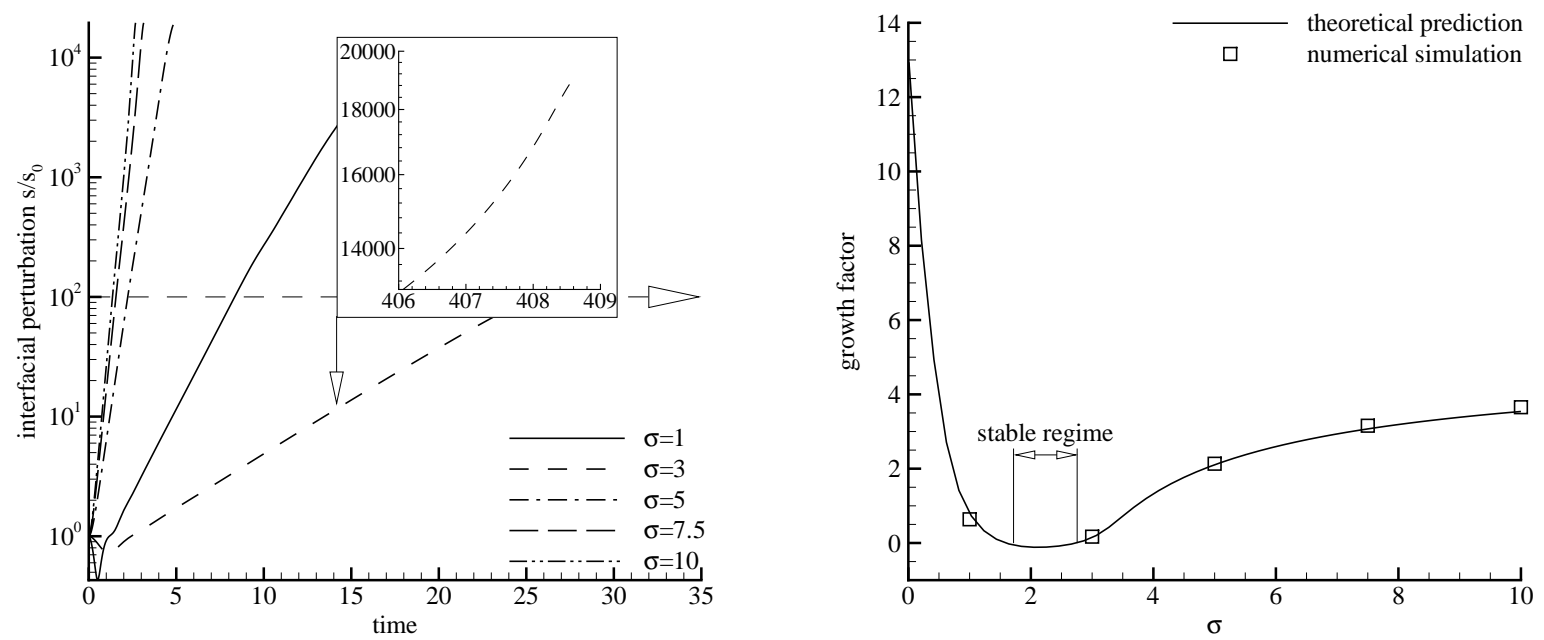

FIGURE 16. Effect of the conductivity ratio $\sigma$ on the interfacial instability in plane two-layer Couette flow. (a) Temporal evolution of the interfacial perturbation $s / s_{0}$ and (b) comparison between the numerically simulated growth rates $a$ and the theoretical predictions of the linear stability theory $c_{i} k$ for a pair of leaky dielectric fluids at $l / h_{1}=2, R e=10, T=0.5, E_{w}=10, \epsilon=5$.

The linear theory predicts a window of stability along this line between $\sigma \approx 1.8$ and $\sigma \approx 2.7$, and we present computations at five different values of $\sigma=1,3,5,7.5$ and 10. Figure 16 shows the evolution of the interfacial perturbation $s / s_{0}$ (a) and the corresponding growth rates (b) - both from the simulations and the linear theory. The simulations and linear theory are in excellent agreement at early times (b) and the size of the growth rates is found to have a non-monotonic behavior with $\sigma$. Note especially the rather slow growth of the perturbation for $\sigma=3$ due to its proximity to the stability window. We note in passing that for $\sigma=3$, higher growth rates have been found in the simulations at smaller permittivity ratios $\epsilon=0.5$ and 1.0 as well as higher permittivity ratios $\epsilon=5,7.5$ and 10 , showing non-monotonicity as $\epsilon$ is varied also. A particularly physically interesting result is the existence of a window of complete stabilisation that is revealed by the results in figure16 (b). It can be seen that there exists a region $1.788 \leq \sigma \leq 2.737$ indicated on the figure, where the interface is linearly stable. Even though this holds for $\epsilon=5$, the complete stabilization window will extend to other neighboring values of $\epsilon$ by continuity. Such linear results are not new and have been discussed in Li et al. (2007), for example.

We turn next to the corresponding spatio-temporal interfacial evolution of the results given in figure 16 . Figure 17 shows such results for the four values $\sigma=1,3,7.5$ and 10 (a) in conjunction with the lower plate 

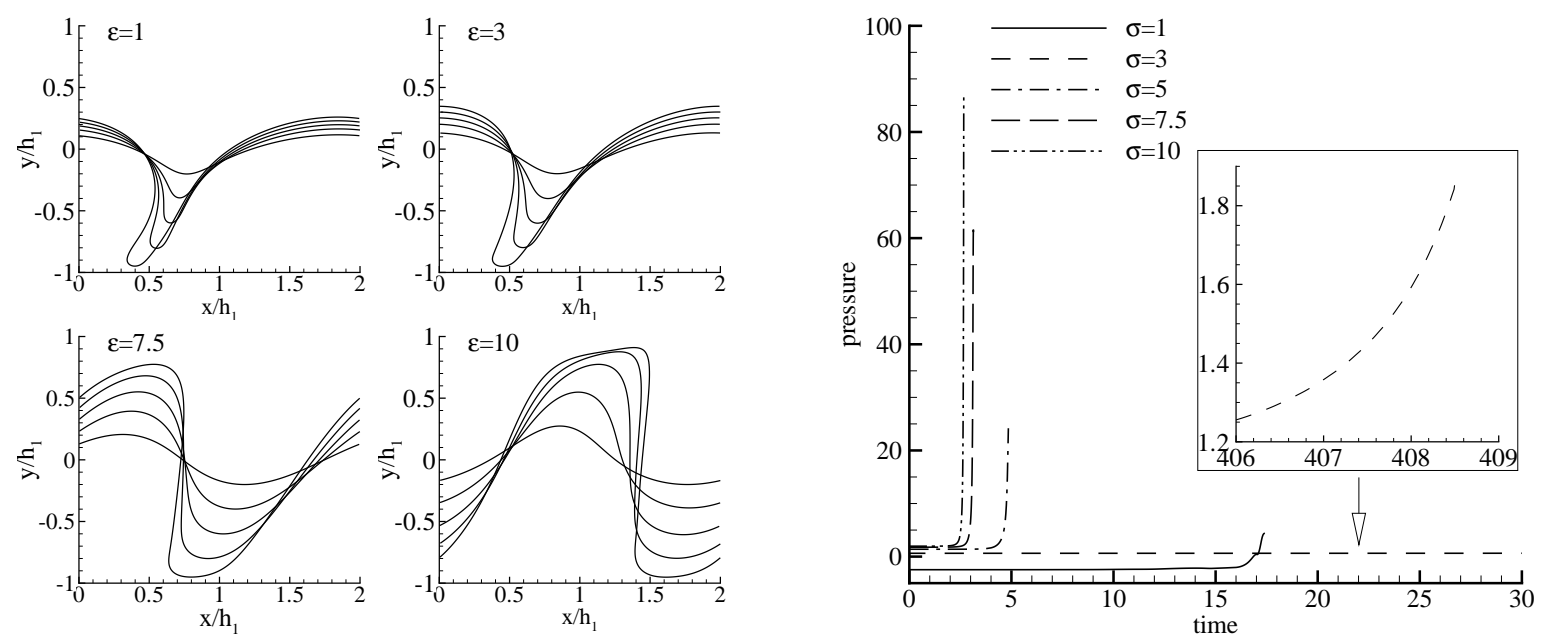

FIGURE 17. Effect of the conductivity ratio $\sigma$ on the interfacial instability in plane two-layer Couette flow. (a) Temporal evolution of the perturbed interface shape and (b) time history of the maximum wall pressure on the lower plate for a pair of leaky dielectric fluids at $l / h_{1}=2, R e=10, T=0.5, E_{w}=10, \epsilon=5$.

maximum pressure evolution (b). For $\sigma=1$, the initially flat interface quickly becomes multi-valued and develops a pair of fingers which propagate opposite to each other and opposite to the shearing direction as seen in the figure. As time progresses a complicated, multi-valued interfacial shape forms which is a result of topological transitions which the code is naturally capable of describing. A striking result is that the horizontal motion of the evolving interface propagates against the local mean-flow direction with the early time finger in the region $y>0$ propagating to the left while that in $y<0$ propagates to the right. Eventually, the interfacial sections appear to slide along the lower plate without approaching it further, while the tip of the finger bends back towards higher vertical positions. The simulations were carried out to times of over 400 time units as shown in the insets and no touchdown was found. The rather slow growth rate predicted by linear stability theory was confirmed by numerical simulation but linear results are quite meaningless in view of the highly complex evolution just described and interfacial pinching that forms blobs of one phase suspended in the other. At larger values of $\sigma$ the behavior changes and the generic non-linear phenomena are much in line with what was found for perfect dielectrics, i.e. finger formation takes place followed by a finite time touchdown of the lower wall. The width of the finger decreases as $\sigma$ increases - compare for example the panel corresponding to $\sigma=3$ with those for $\sigma=7.5$ and 10. Elongated fingers develop and are accompanied by the formation of large fluid reservoirs on either side. We note again that instantaneous interfacial shapes 

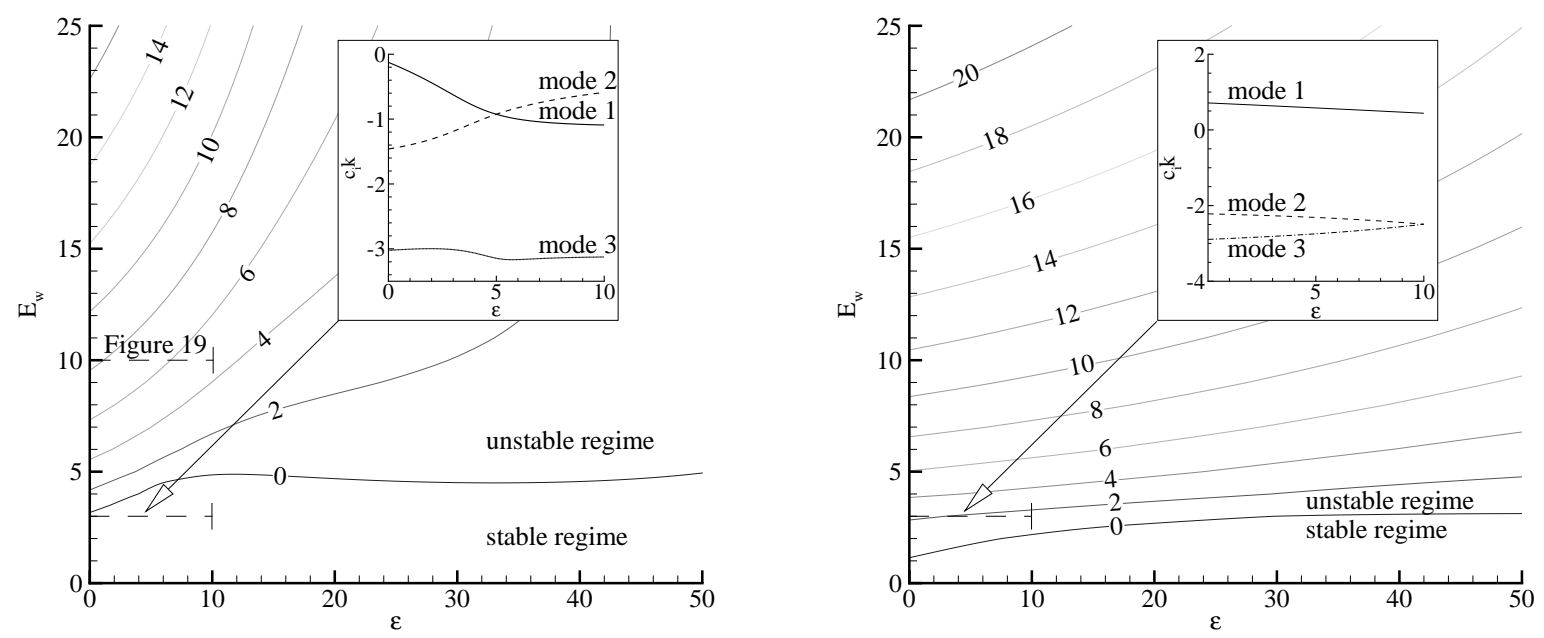

Figure 18. Effect of the permittivity ratio $\epsilon$ on the interfacial instability in plane two-layer Couette flow. Contours of constant growth rate $\left(c_{i} k=\right.$ const.) for (a) $\sigma=5$ and (b) $\sigma=10$ for a pair of leaky dielectric fluids at $l / h_{1}=2, R e=10, T=0.5$.

at $\epsilon=\sigma=5$ are depicted in figure 4 , since the equations for perfect dielectrics are the same as those for leaky dielectrics when $\epsilon=\sigma$. As the growth rates increase with $\sigma$, e.g. $\sigma=10$, this finger forms a drop-like tip connected by a long thinning fluid neck, a finding which is in line with the results discussed in the previous section for the pair of perfect dielectric fluids. The time history of the maximum pressure on the lower plate (figure 4 (b)) also reveals a strong dependence on the growth rate of the perturbed system. For the present set of conductivity ratios the maximum plate pressure at the terminal time of the simulation is found at $\sigma=10$. Interestingly, the maximum pressure at $\sigma=1$ is negative for a wide time range and increases to small positive value at the very end of the simulation (no touchdown is found here).

\subsubsection{Effect of permittivity ratio $\epsilon$}

In this Section we fix $l / h_{1}=2, R e=10, T=0.5$ and present results for two different values $\sigma=5$ and $\sigma=10$ as $\epsilon$ varies. Figure 18 depicts contour lines of constant growth rate $\left(c_{i} k=\right.$ const. $)$ in the $\epsilon$ - $E_{w}$ plane (numbers on the curves denote the value of the corresponding growth rate). The results reveal that the lowest field strength that is required in order to induce interfacial instability at a given growth rate, is found in the limit $\epsilon \rightarrow 0$. For finite permittivity ratios, the curves of constant growth rates increase towards higher field strengths. Moreover, the slopes of the lines $c_{i} k=$ const. become vertical at sufficiently high values of 

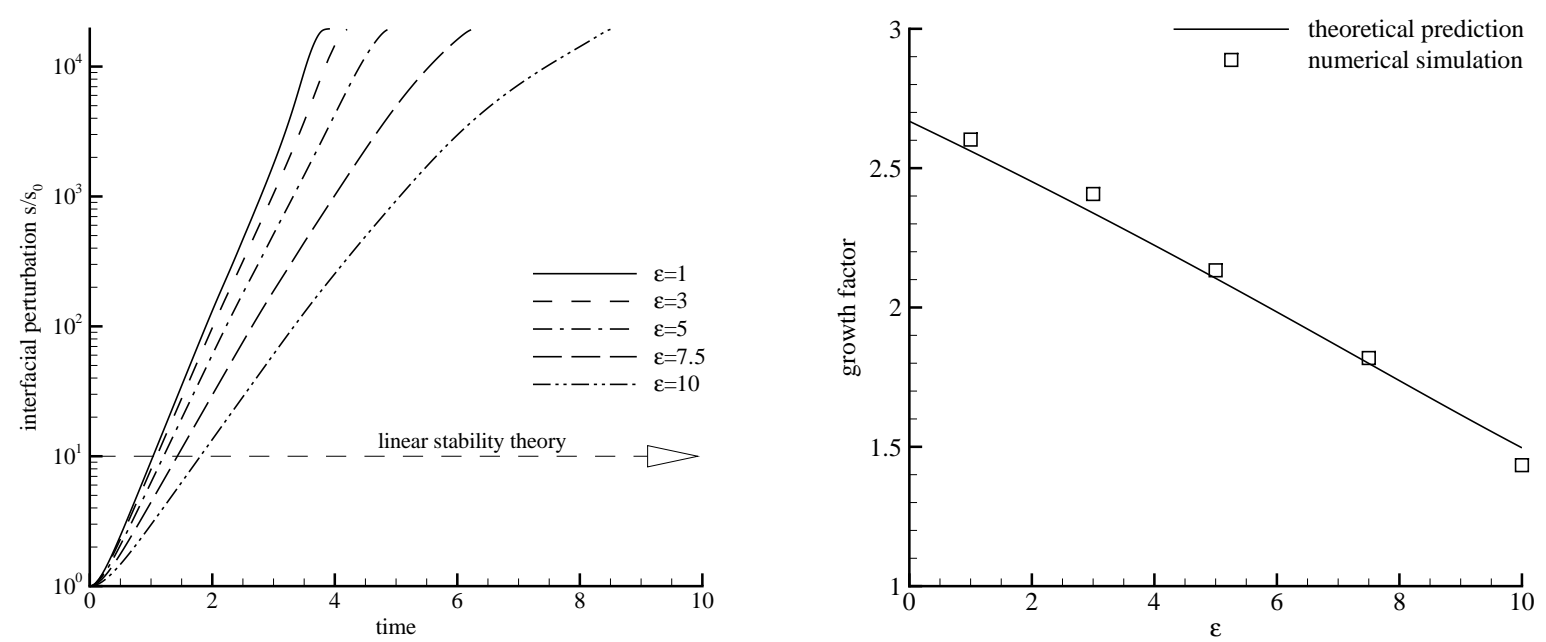

FIGURE 19. Effect of the permittivity ratio $\epsilon$ on the interfacial instability in plane two-layer Couette flow. (a) Temporal evolution of the interfacial perturbation $s / s_{0}$ and (b) comparison between the numerically simulated growth rates $a$ and the predictions of the linear stability theory $c_{i} k$ for a pair of leaky dielectric fluids at $l / h_{1}=2, R e=10, T=0.5, E_{w}=10, \sigma=5$.

$E_{w}$ implying that the interfacial growth rates converge to a constant in the limit of high field strengths. The practical interpretation of this result is that for a pair of leaky dielectrics with fixed permittivity ratio, an increase in the field strength beyond a certain threshold value leads to a saturation instability level. We note also that the curve of marginal stability $\left(c_{i} k=0\right)$ is almost independent of the permittivity ratio. By inspecting the eigenvalue spectrum at $E_{w}=3$ (see the inset of figure 18) we find that there is mode crossing in the stable regime (i.e. $c_{i} k<0$ ) between the two most unstable modes 1 and 2 , implying that mode 2 becomes the dominant unstable mode for $\epsilon>5$. Higher values of $E_{w}$ increase the growth rates of mode 1, while mode 2 becomes stable and crosses with mode 3 instead. Increasing the conductivity ratio to $\sigma=10$ (figure 18 (b)) produces nearly horizontal constant $c_{i} k$ contour lines at low and moderate field strengths (with respect to the chosen range of $E_{w}$ ), which implies a weakened dependence of the growth rate on the permittivity ratio $\epsilon$. The first two least stable modes 2 and 3 cross in the regime of low permittivity ratios, as shown in the insert constructed from the indicated line $E_{w}=3$. This mode crossing also causes a slight decrease of the growth rate of the unstable mode 1 . A crossing of mode 1 with any other stable mode does not occur within the spanned $\epsilon-E_{w}$ space.

In figure 19 we show the effect of $\epsilon$ variations on the interfacial dynamics in leaky dielectric systems, for 

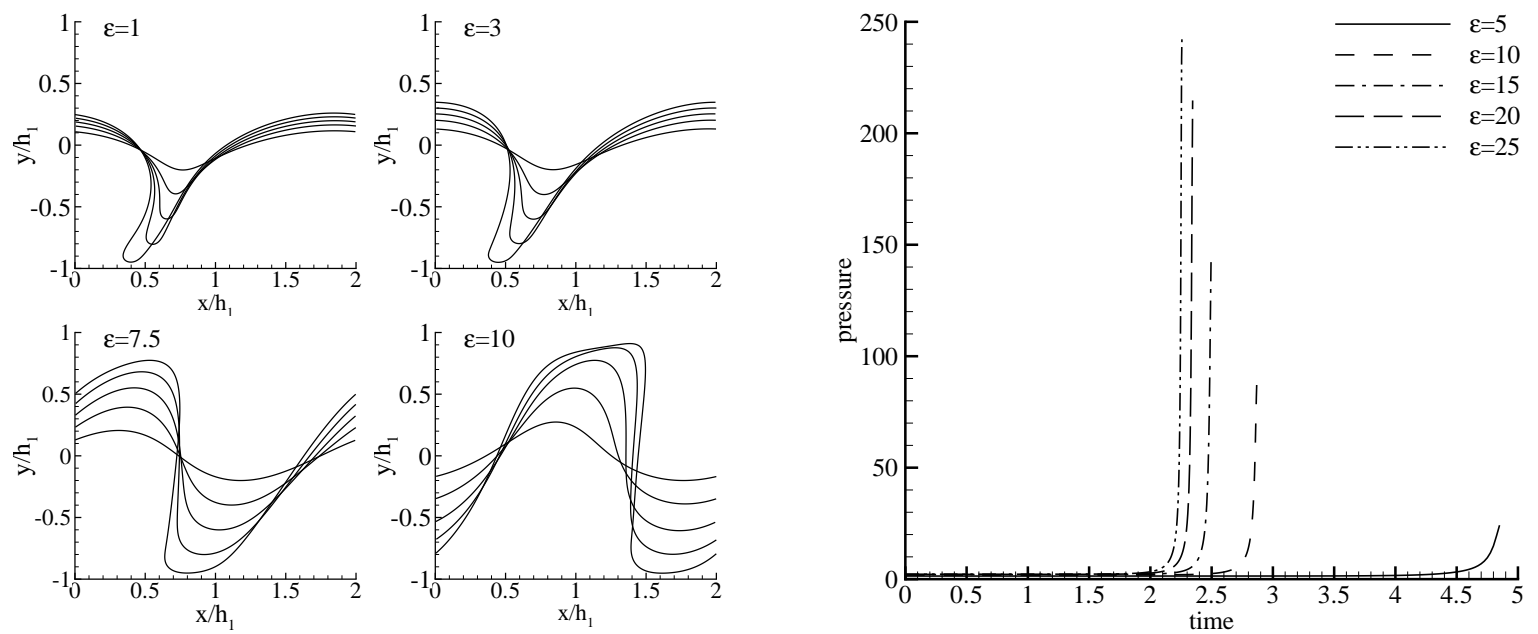

FIGURE 20. Effect of the permittivity ratio $\epsilon$ on the interfacial instability in plane two-layer Couette flow. (a) Temporal evolution of the perturbed interface shape and (b) time history of the maximum pressure on the lower plate for a pair of leaky dielectric fluids at $l / h_{1}=2, R e=10, T=0.5, E_{w}=10, \sigma=5$.

a sequence of permittivity ratios $\epsilon=1,3,5,7.5,10$ and a fixed value of $\sigma=5$, other parameters equal to those above. Panel (a) shows the corresponding evolution of the maximum interfacial amplification $s / s_{0}$, and it can be seen that the exponential linear growth rate found at early times, decreases monotonically as $\epsilon$ increases. This in turn suggests that the terminal touchdown times increase monotonically with $\epsilon$ and this is indeed confirmed by the results. The direct numerical simulation findings are in excellent agreement with the results of linear stability analysis, as the comparison in figure 19 (b) confirms. For a clearer illustration of the instability regime considered here and its position in the phase-space of solutions, we have marked the range of $\epsilon$ values used to produce these results as a dashed line in figure 18 (a).

Our simulations reveal that variations in the permittivity ratio in leaky dielectric systems has a striking effect on the temporal evolution of the perturbed interface. This is quantified in the spatio-temporal evolution of the interface at a fixed conductivity ratio $\sigma=5$ and for a range of $\epsilon$, found in figure 20 (a). At the smaller values of $\epsilon=1$ and 3 , the relatively larger growth rates produce a single interpenetrating finger which is swept by the flow upstream and touches the lower wall in finite time. The width of the finger increases as $\epsilon$ increases from 1 to 3 . Increasing $\epsilon$ to 7.5 and 10 reduces the growth rate and thus causes stronger interaction between the finger region and the bulk fluid. As a result the finger neck widens as is evident from the results for (note that corresponding interfacial shapes for $\epsilon=5$ can be found in figure 4 since $\sigma=\epsilon=5$ here). The 
physics behind the growth rate reductions as the permittivity ratio is increased (as opposed to an increase in instability for dielectric fluid pairs) is due to the induction of tangential electrical stresses which couple to affect the flow in the bulk and hence the stability characteristics - see also the detailed linear stability study of Li et al. (2007). The results also reveal an interesting and distinct non-linear phenomenon for the fingering instability at $\epsilon=7.5$ and 10 as compared lower values of $\epsilon$. The phenomenon is most striking at $\epsilon=10$ where it is seen that the disturbance evolves as a large amplitude non-linear wave whose maximum is driven towards the upper wall and its minimum towards the lower wall (here we link the term non-linear to the growth rate of the developing instability and not its shape and note that the growth rate depends on the amplitude since the wave is non-linear). Touchdown is almost simultaneous and in the computations for the present set of parameters it takes place at the lower wall first. Due to the horizontal periodicity of the flow, and allowing for a topological transition and contact line motions, we can surmise that the upper part of the interface which is already in close proximity to the upper wall, will touch the latter thus producing an alternating periodic series of plug flows of fluid 1 follows by fluid 2 . This is reminiscent of the phenomenon described in the experiments of Ozen et al. (2006a) where a monodisperse distribution of encapsulated droplets was produced using EHD instabilities in micro-channels, but additional parameter studies are needed to quantify the observations of our numerical experiments. We note that in regimes that do not evolve with this canonical type of large amplitude undulations, a penetrating finger forms instead which touches the lower plate (for our set of parameters) in finite time. In such cases the ultimate droplet formation and fluid separation is quite different from that described in Ozen et al. (2006a). For completeness we include in figure 20 (b) the effect of the permittivity ratio on the time history of the maximum pressure on the lower plate. The maximum value decreases with the permittivity ratio and this is clearly attributed to the reduction of both the growth rate and the tip velocity of the finger instability. Moreover, since the finger tip radius also increases, the sharp peak of the temporal pressure distribution flattens out to a smoother variation. 


\section{Conclusions}

Interfacial instabilities in electrified plane Couette flow have been studied using a combination of numerical simulation and analytical work. Electric fields introduce additional stresses and can modify both the normal and tangential stress balances thus affecting the system's stability and ultimate non-linear dynamics. The base state is unstable to disturbances with wavelengths for which the electrostatic force overcomes the surface tension. At small amplitude linear stability theory provides growth rates arising from a normal mode analysis of the equations and solution of an allied eigenvalue problem. The inclusion of electric fields yields a generalized eigenvalue problem that resembles the two-layer Orr-Sommerfeld equation and this has been solved to determine the growth rates and eigenfunctions of the most unstable modes (see Section 2.2). The linear theory has been followed by numerical simulations of the interfacial dynamics. The applied fronttracking method embeds the fluid interface in the bulk flow region through a narrow region with variable properties and a localized force arising from the presence of surface tension and electrostatic effects at the interface.

Given the large number of parameters and the complexity of the full scale simulations, the simpler case of density and viscosity matched fluids with equal unperturbed fluid layer thicknesses has been considered. A Couette profile was generated by moving the parallel plates confining the fluids at equal speeds and in opposite directions. In addition, an electric field was applied in the cavity by imposing a constant potential difference between the horizontal plates confining the fluids. The transient simulations were started from the analytic velocity field with zero perturbation velocity as an exact solution of the Navier-Stokes equations, by imposing a small-amplitude disturbance onto the initially flat interface. The initial deformation causes perturbations to grow on the interface at different rates and wavelengths. In addition, fluid motion ensues and generates patterns in the two-fluid system with the length of periodicity set by the characteristic length of the most unstable mode.

By comparing transient numerical data with the results of the linear theory we have shown that exponential growth dominates the evolution of the perturbed interface over a wide time scale. Moreover, by tracking the evolution we show that the elementary structures that typically emerge from the perturbed interface are single or multiple fingers of interpenetrating fluid regions. The number and shape of these fingers were found 
to mainly depend on the growth rate of the dominant instability mode in the system. For weakly unstable systems a single, slowly growing fingering instability develops on the interface. At higher growth rates this finger narrows to a drop-like tip connected by a long thinning fluid neck. The invading front induces fluid motion in its neighborhood followed by the formation of lateral bulk reservoirs. Eventually, at highly unstable conditions, this fingering process yields multiple generations of splitting leading to a very ramified interface. In almost all cases the penetrating fingers touch one of the walls in finite time, whereas the wall pressure tends to approach a singular value at touchdown. The numerical simulations have revealed a rich array of dynamical behavior that arises from the present model of electrified two-layer flow. In line with the findings of other authors, we have shown that the presence of surface tension in such system causes the formation of distinct regions of interpenetrating fluids instead of interfacial roll up into a structure like the classical Kelvin-Helmholtz spiral. Inertia was found to enhance the instability of the system but does not alter the topology of the evolving interface in the considered parameter range.

The computational tools described here have been applied to both perfect and leaky dielectric immiscible fluid pairs. The numerical simulations confirm the theoretical finding that a vertical electric field destabilizes the perturbed interface between a pair of perfect dielectrics, irrespective of the permittivity ratio. Increasing the strength of the electric field enhances the stress imbalance at the interface implying faster growth rates and stronger elongated fingers. At even higher field strengths localized dimples originate on the interface, which are quickly magnified into sets of interpenetrating fingers. The time to touchdown decreases as the permittivity ratio increases. By contract, the same electric field can either completely stabilize a system of leaky dielectrics or even further enhance its instability depending on the particular permittivity and conductivity ratio between the fluid pair. Moreover, by analyzing the linear eigenvalue spectrum of leaky dielectrics it was found that the existence of a window of complete stability in parameter space, was caused by the interaction of the most unstable modes in the system. In particular we have computed cases that produce flows with interfacial pinching but no touchdown so that blobs of one fluid are encapsulated in the other - our algorithm is capable of following solutions beyond a topological transition (see figure 17 for example). Our computations also demonstrate that the width of interpenetrating fingers can be controlled for leaky dielectrics: (i) a decrease in width is achieved by an increase in $\sigma$ for fixed $\epsilon$ (figure 17); (ii) an increase 
in width is achieved by increasing $\epsilon$ for a fixed $\sigma$ (figure 20). The latter results indicate another phenomenon that could be useful in practical applications. For the example case $\epsilon=10, \sigma=5$ (other parameters fixed as shown in figure 2010 a wide finger forms an an almost simultaneous touchdown of the upper and lower electrodes is found, thus opening the way of using such systems to separate the two fluids into a alternating train of fluid plugs. Such a mechanism is reminiscent of the monodisperse drop formation experiments of Ozen et al. (2006a) and could find applications in microfluidics - see Song, Chen \& Ismagilov (2006). We note that the train of liquid plugs relaxes to a train of suspended droplets when it leaves the electrified region. Additional computations at the precise experimental conditions are required and are the subject of ongoing work by the authors.

This work was supported by a research grant from the Deutsche Forschungsgemeinschaft. The work of DTP was partly supported by grant DMS-0072228 from the National Science Foundation.

\section{REFERENCES}

Anderson, D. M., McFadden, G. B. \& Wheeler, A. A. 1998 Diffuse-interface methods in fluid mechanics. Ann. Rev. Fluid Mech. 30, 139-165.

BAygents, J. C. \& BAldessari, F. 1998 Electrohydrodynamic instability in a thin uid layer with an electrical conductivity gradient. Phys. Fluids 10, 301-311.

Canuto, C., Hussaini, M. Y., Quarteroni, A. \& Zang, T. 1988 Spectral methods in fluid dynamics. Springer, New York.

Charles, M. E., Govier, G. W. \& Hodgson, G. W. 1961 The horizontal pipeline flow of equal density oil-water mixtures. Can. J. Chem. Eng. 39, 27-36.

Chen, C. H., Lin H. Lele S. K. \& Santiago, J. G. 2005 Convective and absolute electrokinetic instability with conductivity gradients. J. Fluid Mech. 524, 263-303.

Craster, R. V. \& Matar, O. K. 2005 Electrically induced pattern formation in thin leaky dielectric films. Phys. Fluids 17 (32104-1).

Dongarra, J. J., Straughan, B. \& Walker, D. W. 1996 Chebyshev tau-QZ algorithm methods for calculating spectra of hydrodynamics stability problems. Appl. Num. Math. 22, 399-434.

Du, J., Fix, B., Glimm, J., JiA, X., Li, X., Li, Y. \& Wu, L. 2005 A simple package for front tracking. J. Comput. Phys. 213 (2), 613-628. 
El Moctar, A. O., Aubry, N. \& Batton, J. 2003 Electro-hydrodynamic microfluidic mixer. Lab Chip 3, $273-280$.

Glimm, J., Grove, J.W., W., J., Li, X. L., Shyue, K.-M., Zhang, Q. \& Zeng, Y. 1998 Three-dimensional front tracking. SIAM J. Sci. Comput. 19, 703-727.

Hagerdon, J. G., Martyn, N. S. \& Douglas, J. F. 2004 Breakup of a uid thread in a conned geometry: dropletplug transition, perturbation sensitivity, and kinetic stabilization with connement. Phys. Rev. E 69, 056312.

Hirt, C. W. \& Nichols, B. D. 1981 Volume of fluid (vof) method for the dynamics of free boundaries. J. Comput. Phys. 39, 201-225.

Hooper, A. P. 1989 The stability of two superposed viscous fluids in a channel. Phys. Fluids A 1 (7), 1133-1142.

JACKson, J.D. 1962 Classical Electrodynamics. John Wiley \& Sons, New York.

Landau, L. D. \& Lifshitz, E. M. 1960 Electrodynamics of continuous media. Oxford: Pergamon.

LeE, L. \& LeVeque, R. J. 2003 An immersed interface method for incompressible Navier-Stokes equations. SIAM J. Numer. Anal. 25 (832-856).

LeVeque, R. J. \& LI, Z. 1994 The immersed interface method for elliptic equations with discontinuous coefficients and singular sources. SIAM J. Numer. Anal. 13, 1019-1044.

Li, F., Ozen, O., Aubry, N., Papageorgiou, D. T. \& Petropoulos, P. G. 2007 Linear stability of a two-fluid interface for electrohydrodynamic mixing. J. Fluid Mech. 583, 347-377.

Lin, H. 2004 Electrokinetic instability in microchannel flows: A review. Mec 16, 1922-1935.

Lin, H., Storey B. D. Oddy M. H. Chen C. H. \& Santiago, J.G. 2004 Instability of electrokinetic microchannel flows with conductivity gradients. Phys. Fluids 16, 1922-1935.

Mählmann, S. \& Papageorgiou, D.T. 2009 Numerical study of electric field effects on the deformation of twodimensional liquid drops in simple shear flow at arbitrary reynolds number. J. Fluid Mech. 626, 367-393.

Melcher, J. R. \& Schwarz, JR, W. J. 1968 Interfacial relaxation overinstability in a tangential electric field. Phys. Fluids 11 (12), 2604-2616.

Melcher, J. R \& Smith, JR, C. V. 1969 Electrohydrodynamic charge relaxation and interfacial perpendicular-field instability. Phys. Fluids 12 (4), 778-790.

Navaneetham, G. \& Posner, J. D. 2009 Electrokinetic instabilities of non-dilute colloidal suspensions. J. Fluid Mech. 619, 331-365.

Osher, S. \& Sethina, J. 1988 Front propagating with curvature dependent speed: Algorithms based on HamiltonJacobi formulations. J. Comput. Phys. 79, 12-49.

Papageorgiou, D. T. \& Vanden-Broeck, J.-M. 2004a Antisymmetric capillary waves in electrified fluid sheets. European Journal of Applied Mathematics 15 (6), 609-623. 
Papageorgiou, D. T. \& Vanden-Broeck, J.-M. $2004 b$ Large amplitude capillary waves in electrified fluid sheets. J. Fluid Mech. 508, 71-88.

Peskin, C. S. 1988 Numerical analysis of blood flow in the heart. J. Comput. Phys. 25, 220-252.

Peskin, C. S. 2002 The immersed boundary method. Acta Numerica 11, 1-39.

Posner, J. D. \& Santiago, J.G. 2006 Convective instability of electrokinetic flows in a cross-shaped microchannel. J. Fluid Mech. 555, 1-42.

PozRikidis, C. S. 2001 Interfacial dynamics for Stokes flow. J. Comput. Phys. 169, 250-301.

RenARdy, Y. 1987 The thin layer effect and interfacial stability in a two-layer Couette flow with similar liquids. Phys. Fluids 30 (6), 1627-1637.

Saville, D. A. 1997 Electrohydrodyamics: the Taylor-Melcher dielectric model. Annu. Rev. Fluid Mech. 29, 27-64.

Shin, S. \& Juric, D. 2002 Modeling three-dimensional multiphase flow using a level contour reconstruction method for front tracking without connectivity. J. Comput. Phys. 180, 427-470.

Song, H., Chen, D.L. \& Ismagilov, R.F. 2006 Reactions in droplets in microfluidic channels. Angewadte Chimie $45(7336-7356)$.

Thaokar, R. M. \& Kumaran, V. 2005 Electrohydrodynamic instability of the interface between two fluids confined in a channel. Phys. Fluids 17 (8), 84104-1.

Tomar, G., Gerlach, D., Biswas, G., Alleborn, N., Sharma, A., Durst, F., Welch, S. W. J. \& Delgado, A. 2007 Two-phase electrohydrodynamic simulations using a volume-of-fluid approach. J. Comp. Phys. 227, $1267-1285$.

Trefethen, L. N. 2000 Spectral Methods in MATLAB. SIAM, Philadelphia.

Tseluiko, D., Blyth, M. G., Papageorgiou, D. T. \& Vanden-Broeck, J. M. 2008 Effect of an electric eld on $\operatorname{lm}$ ow down a corrugated wall at zero reynolds number. Phys. Fluids 20, 042103.

Uguz, A. K. \& Aubry, N. 2008 Quantifying the linear stability of a flowing electrified two-fluid layer in a channel for fast electric times for normal and parallel electric fields. Phys. Fluids 20 (092103).

Uguz, A. K., Ozen, O. \& Aubry, N. 2008 Electric field effect on a two-fluid interface instability in channel flow for fast electric times. Phys. Fluids 20 (031702).

Unverdi, O. \& Tryggvason, G. 1992 A front-tracking method for viscous incompressible, multi-fluid flows. J. Comp. Phys. 100, 25-37.

Yiantsios, S. G., Brian, G. \& Higgins, G. 1988 Linear stability of plane Poiseuille flow of two superposed fluids. Phys. Fluids 31 (11), 3225-3238.

YıH, C.S 1967 Instability due to viscosity stratification. J. Fluid Mech. 27, 337-352. 